\title{
RANDOM TIME-CHANGE WITH INVERSES OF MULTIVARIATE SUBORDINATORS: GOVERNING EQUATIONS AND FRACTIONAL DYNAMICS
}

\author{
LUISA BEGHIN*, CLAUDIO MACCI**, AND COSTANTINO RICCIUTI*
}

\begin{abstract}
It is well-known that compositions of Markov processes with inverse subordinators are governed by integro-differential equations of generalized fractional type. This kind of processes are of wide interest in statistical physics as they are connected to anomalous diffusions. In this paper we consider a generalization; more precisely we mean componentwise compositions of $\mathbb{R}^{d}$-valued Markov processes with the components of an independent multivariate inverse subordinator. As a possible application, we present a model of anomalous diffusion in anisotropic medium, which is obtained as a weak limit of suitable continuous-time random walks.
\end{abstract}

\section{Contents}

1. Introduction

2. Bivariate subordinators 3

2.1. Preliminaries 4

2.2. Some results 7

3. Inverses of bivariate subordinators 9

3.1. Distributional properties 9

3.2. Governing equation 13

4. Time-change of bivariate Markov processes 14

4.1. Time-change of continuous-time Markov chains. 16

5. CTRW limits and anomalous diffusion in anisotropic media 18

5.1. Functional limit results $\quad 19$

References $\quad 22$

Date: May 13, 2020.

2010 Mathematics Subject Classification. 60G52, 60G22, 60F99, 60K40.

Key words and phrases. Random time-change, multivariate Lévy processes, subordinators, anomalous diffusion, continuous time random walks, fractional operators.

* Dipartimento di Scienze Statistiche, Università di Roma Sapienza, Piazzale Aldo Moro 5, 00185, Roma, Italy

** Dipartimento Matematica, Università di Roma Tor Vergata, viale della Ricerca Scientifica 1, 00133, Roma, Italy . 


\section{INTRODUCTION}

A multivariate subordinator is a $\mathbb{R}^{d}$-valued Lévy process with non-decreasing (possibly dependent) marginal components. In [1], the authors studied the subordinated process $\left(X_{1}\left(H_{1}(t)\right), \ldots, X_{d}\left(H_{d}(t)\right)\right)$, where $\left(X_{1}(t), \ldots, X_{d}(t)\right)$ is a timehomogeneous $\mathbb{R}^{d}$-valued Markov process and $\left(H_{1}(t), \ldots, H_{d}(t)\right)$ is an independent multivariate subordinator. This generalized the well-known case of a common random time $H(t)$ for all the components (for further developments consult [39], [40], [41]). Time-changes of Markov processes with multivariate subordinators also appear in some references in the literature with applications to finance; see e.g. [24].

In the same spirit, this paper focuses on the time-changed process

$$
\left(X_{1}\left(L_{1}(t)\right), \ldots, X_{d}\left(L_{d}(t)\right)\right), \quad t \geq 0
$$

where $\left(X_{1}(t), \ldots, X_{d}(t)\right)$ is a $\mathbb{R}^{d}$-valued Markov process, while, for each $j=1, \ldots, d$, $L_{j}(t)$ is the inverse (or right-continuous hitting time) of $H_{j}(t)$, i.e.

$$
L_{j}(t)=\inf \left\{x>0: H_{j}(x)>t\right\},
$$

which is assumed to be independent of $\left(X_{1}(t), \ldots, X_{d}(t)\right)$. Without loss of generality, we assume that $\left(X_{1}(0), \ldots, X_{d}(0)\right)=(0, \ldots, 0)$ almost surely.

The literature inspiring our study is vast. Indeed many works, such as [2], [19], [34], [32], [33], [35], [36], concern processes of the form

$$
\left(X_{1}(L(t)), \ldots, X_{d}(L(t)), \quad t \geq 0,\right.
$$

where each component of the original process is time-changed by the same random time $L(t)=\inf \{x>0: H(x)>t\}$, namely the inverse of the same univariate subordinator.

There exists a well established theory for the stochastic process in (1.2). An interesting fact is that the density $p(x, t)$ of $(1.2), x \in \mathbb{R}^{d}$, is governed by integrodifferential equations of the form

$$
\mathcal{D}_{t} p(x, t)=\mathcal{G} p(x, t), \quad x \neq 0,
$$

where $\mathcal{D}_{t}$ is the operator defined by

$$
\mathcal{D}_{t} h(t):=\int_{0}^{\infty}(h(t)-h(t-\tau)) \nu(d \tau)
$$

$\nu$ is the Lévy measure of the underlying subordinator $H(t)$ and $\mathcal{G}$ is the dual to the generator of $\left(X_{1}(t), \ldots, X_{d}(t)\right)$. The operator $\mathcal{D}_{t}$ is called generalized fractional derivative because, when $L(t)$ is an inverse stable subordinator of index $\alpha \in(0,1)$, it reduces to the Caputo fractional derivative

$$
\frac{d^{\alpha}}{d t^{\alpha}} f(t)=\int_{0}^{\infty} \frac{d}{d \tau} f(\tau) \frac{(t-\tau)^{-\alpha-1}}{\Gamma(1-\alpha)} d \tau
$$

(see e.g. [18] p. 92 for details). Moreover, if $\left(X_{1}(t), \ldots, X_{d}(t)\right)$ is a Lévy process, then processes defined in (1.2) can be seen as scaling limits of suitable continuous time random walks (hereafter CTRW). We recall that a CTRW is defined by a sequence of i.i.d. jumps $Y_{i} \in \mathbb{R}^{d}$ separated by i.i.d. inter-arrival times $J_{i} \in \mathbb{R}^{+}$.

In the special case where $\left(X_{1}(t), \ldots, X_{d}(t)\right)$ is a Brownian motion and $L(t)$ is the inverse of a stable subordinator, then the process (1.2) is a so-called subdiffusion, which has great interest in many areas of statistical physics (on this point, consult e.g. [26], [27] and [37]). See also [20] and [21], for the more general case where the external process is the fractional Brownian motion. Basically, a subdiffusion 
models the case where the particle is subject to a trapping effect which delays the motion with respect to the simple Brownian process; clearly, in this model it is assumed that the trapping effect is the same in all directions (the external medium is isotropic) and thus the time-changed process is isotropic as well as the Brownian motion. It is natural, then, to ask what happens if the motion of the particle takes place in an anisotropic medium, where the trapping effect is different depending on the direction. This is the strongest physical argument that inspired our investigation of processes in (1.1).

To our knowledge, the study of the model (1.1) is a completely new problem. In this paper we restrict our analysis to the case $d=2$. Our choice is motivated by technical problems; indeed, some of the results presented in this paper hold only for $d=2$, and, moreover, some calculations are quite cumbersome even with this restriction. However, we are sure that the discussion of the 2-dimensional case, besides making the present exposure clearer, will be useful for possible future studies of the multidimensional counterpart.

Now we give a brief description of the main results and the outline of the paper. In Section 2 we recall the definition of bivariate subordinators and we find some auxiliary results. A crucial part of the paper is Section 3, where we study in depth some distributional properties and a governing equation of the biparameter process $\left(L_{1}\left(t_{1}\right), L_{2}\left(t_{2}\right)\right)$; in this way we present an extension of the well-known theory of inverse subordinators to the 2-dimensional case. In Section 4 we focus on the biparameter process $\left(X_{1}\left(L_{1}\left(t_{1}\right)\right), X_{2}\left(L_{2}\left(t_{2}\right)\right)\right)$ and we prove that its density $p\left(x_{1}, x_{2}, t_{1}, t_{2}\right)$ solves an equation of the following form

$$
\mathcal{D}_{t_{1}, t_{2}} p\left(x_{1}, x_{2}, t_{1}, t_{2}\right)=\mathcal{G} p\left(x_{1}, x_{2}, t_{1}, t_{2}\right), \quad x_{1}, x_{2} \neq 0,
$$

where

$$
\mathcal{D}_{t_{1}, t_{2}} h\left(t_{1}, t_{2}\right):=\int_{0}^{\infty} \int_{0}^{\infty}\left(h\left(t_{1}, t_{2}\right)-h\left(t_{1}-\tau_{1}, t_{2}-\tau_{2}\right)\right) \phi\left(d \tau_{1}, d \tau_{2}\right),
$$

$\phi$ is the Lévy measure of the underlying bi-dimensional subordinator $\left(H_{1}\left(t_{1}\right), H_{2}\left(t_{2}\right)\right)$ and $\mathcal{G}$ is the dual to the generator of $\left(X_{1}(t), X_{2}(t)\right)$. The operator $\mathcal{D}_{t_{1}, t_{2}}$ in (1.6) is a bi-dimensional version of the generalized fractional derivative appearing in (1.3); it was already introduced in [30] in advection-dispersion equations governing multidimensional stable Lévy motions, though acting on the space variables.

Finally, in Section 5, we focus on the special case where $\left(X_{1}(t), X_{2}(t)\right)$ is a Brownian motion $\left(B_{1}(t), B_{2}(t)\right)$, while $\left.\left(L_{1}\left(t_{1}\right)\right), L_{2}\left(t_{2}\right)\right)$ is the inverse of a bivariate stable subordinator. In particular, we illustrate how to construct a CTRW converging to $\left(B_{1}\left(L_{1}(t)\right), B_{2}\left(L_{2}(t)\right)\right)$ under a suitable scaling limit.

Notation: For simplicity, throughout the paper we will often denote a process $\{X(t), t \geq 0\}$ by $X(t)$; moreover, we will often denote a bi-paramenter process $\left\{\left(X\left(t_{1}\right), Y\left(t_{2}\right)\right), t_{1} \geq 0, t_{2} \geq 0\right\}$ by $\left(X\left(t_{1}\right), Y\left(t_{2}\right)\right)$.

\section{Bivariate SUbordinators}

This section is devoted to bivariate subordinators in the sense of [1]. In Subsection 2.1, we review some known facts and we connect them to the general theory of multivariate Lévy processes and their governing equation (see [34], Chapt. 6, and also [11] and [30] for the special case of multivariate stable processes). Then, in Subsection 2.2 we will present some original results. 
2.1. Preliminaries. A Lévy process $\left\{\left(H_{1}(t), H_{2}(t)\right), t \geq 0\right\}$ is said to be a bivariate subordinator if its components are both a.s. non-decreasing; we restrict our attention to the case of pure jump subordinators, having no drift. The characteristic function of $\left(H_{1}(t), H_{2}(t)\right)$ can be written (see [1]) as

$$
\mathbb{E} e^{i\left(\alpha H_{1}(t)+\beta H_{2}(t)\right)}=e^{t \sigma(\alpha, \beta)}, \quad \alpha, \beta \in \mathbb{R},
$$

where

$$
\sigma(\alpha, \beta)=\iint_{\mathbb{R}_{2}^{+}}\left(e^{i\left(\alpha x_{1}+\beta x_{2}\right)}-1\right) \phi\left(d x_{1}, d x_{2}\right)
$$

and $\phi\left(d x_{1}, d x_{2}\right)$ denotes the Lévy measure; thus $\phi$ is a measure on $\mathbb{R}_{+}^{2}=\left\{\left(x_{1}, x_{2}\right) \in\right.$ $\left.\mathbb{R}^{2}: x_{1} \geq 0, x_{2} \geq 0\right\}$ such that

$$
\iint_{\mathbb{R}_{2}^{+}} \min \left(\sqrt{x_{1}^{2}+x_{2}^{2}}, 1\right) \phi\left(d x_{1}, d x_{2}\right)<\infty
$$

An equivalent formulation can be given in terms of the Laplace transforms, i.e.

$$
\mathbb{E} e^{-\eta_{1} H_{1}(t)-\eta_{2} H_{2}(t)}=e^{-t S\left(\eta_{1}, \eta_{2}\right)} \quad \eta_{1}, \eta_{2} \geq 0,
$$

where

$$
S\left(\eta_{1}, \eta_{2}\right)=\iint_{\mathbb{R}_{2}^{+}}\left(1-e^{-\eta_{1} x_{1}-\eta_{2} x_{2}}\right) \phi\left(d x_{1}, d x_{2}\right)
$$

is a bivariate Bernstein function (in the sense of [8], Chapt. 4). The marginal processes are univariate subordinators, hence

$$
\mathbb{E} e^{-\eta_{k} H_{k}(t)}=e^{-t T_{k}\left(\eta_{k}\right)} \quad \eta_{k} \geq 0 \quad k=1,2,
$$

where

$$
T_{k}\left(\eta_{k}\right)=\int_{0}^{\infty}\left(1-e^{-\eta_{k} x}\right) \nu_{k}(d x)
$$

is a Bernstein function and $\nu_{k}$ is the Lévy measure associated to $H_{k}$. Throughout this paper we always assume that both $H_{1}$ and $H_{2}$ have infinite activity, namely

$$
\nu_{k}[0, \infty)=\infty \quad k=1,2 .
$$

It is clear that, if $H_{1}$ and $H_{2}$ are independent, then the Lévy measure $\phi$ is supported on the coordinate axes, i.e. it has the form

$$
\phi\left(d x_{1}, d x_{2}\right)=\nu_{1}\left(d x_{1}\right) \delta_{0}\left(d x_{2}\right)+\nu_{2}\left(d x_{2}\right) \delta_{0}\left(d x_{1}\right),
$$

$\delta_{0}$ denoting the Dirac delta measure; then

$$
S\left(\eta_{1}, \eta_{2}\right)=T_{1}\left(\eta_{1}\right)+T_{2}\left(\eta_{2}\right) .
$$

Remark 2.1. In view of some possible applications, we briefly recall some connections with the theory of copulas. It is well-known that a copula allows to separate the dependence structure of a random vector from its univariate marginal distributions. Moreover, since $\phi$ is a measure, it is possible to define a suitable notion of copula, that is the Lévy copula; see e.g. Section 3 in [15] for its definition and basic properties (in particular Theorem 3.6. in [15] can be considered as the analogue of the Sklar's Theorem for Lévy copulas). Lévy copulas characterize the dependence among components of vector valued Lévy processes and they are also used in some estimation problems (see e.g. [12] and [13]). 
In general, if $\left(H_{1}(t), H_{2}(t)\right)$ has joint density $q_{*}\left(x_{1}, x_{2}, t\right)$, then (see [34], Chapt. $6])$ the following equation holds

$$
\frac{\partial}{\partial t} q_{*}\left(x_{1}, x_{2}, t\right)=-\mathcal{D}_{x_{1}, x_{2}} q_{*}\left(x_{1}, x_{2}, t\right),
$$

where

$$
\mathcal{D}_{x_{1}, x_{2}} h\left(x_{1}, x_{2}\right):=\iint_{R_{+}^{2}}\left(h\left(x_{1}, x_{2}\right)-h\left(x_{1}-y_{1}, x_{2}-y_{2}\right)\right) \phi\left(d y_{1}, d y_{2}\right)
$$

on a suitable class of functions $h$. The operator (2.10) can also be defined by means of its Laplace symbol:

$$
\int_{0}^{\infty} \int_{0}^{\infty} e^{-\eta_{1} x_{1}-\eta_{2} x_{2}} \mathcal{D}_{x_{1}, x_{2}} f\left(x_{1}, x_{2}\right) d x_{1} d x_{2}=S\left(\eta_{1}, \eta_{2}\right) \hat{f}\left(\eta_{1}, \eta_{2}\right)
$$

where $\hat{f}\left(\eta_{1}, \eta_{2}\right):=\int_{0}^{\infty} \int_{0}^{\infty} e^{-\eta_{1} x_{1}-\eta_{2} x_{2}} f\left(x_{1}, x_{2}\right) d x_{1} d x_{2}$ and $S$ has been defined in (2.4). Equation (2.9) comes up in the more general setting of Proposition 2.5 below.

The above facts extend the best known results holding for univariate subordinators; indeed, for each $i=1,2$, the marginal density $q_{i}(x, t)$ of $H_{i}(t)$ solves

$$
\frac{\partial}{\partial t} q_{i}(x, t)=-\mathcal{D}_{x}^{(i)} q_{i}(x, t),
$$

where the operator on the right-hand side is

$$
\mathcal{D}_{x}^{(i)} h(x)=\int_{0}^{\infty}(h(x)-h(x-y)) \nu_{i}(d y),
$$

whose Laplace symbol is defined by

$$
\int_{0}^{\infty} e^{-\eta x} \mathcal{D}_{x}^{(i)} h(x) d x=T_{i}(\eta) \tilde{h}(\eta)
$$

Now we present two examples of bivariate subordinators.

Example 2.2. Let $\left(H_{1}^{\alpha}(t), H_{2}^{\alpha}(t)\right)$ be a bivariate stable subordinator of index $\alpha \in$ $(0,1)$, i.e. a bivariate stable process with non decreasing components. Its Lévy measure can be expressed in polar coordinates as

$$
\phi_{\alpha}(d r, d \theta)=\frac{C \alpha}{r^{1+\alpha}} d r M(d \theta) \quad 0 \leq \theta \leq \frac{\pi}{2},
$$

where $M(d \theta)$ is a measure on the arc of circle $\left\{(x, y) \in \mathbb{R}_{+}^{2}: x^{2}+y^{2}=1\right\}$. If $H_{1}^{\alpha}(t)$ and $H_{2}^{\alpha}(t)$ are independent, we can write

$$
M(d \theta)=p \delta_{0}(d \theta)+(1-p) \delta_{\frac{\pi}{2}}(d \theta)
$$

for some $p \in(0,1)$, where $\delta_{x}$ denotes the Dirac delta in $x$.

By simple calculations we see that the characteristic exponent (2.2) has the form

$$
\begin{aligned}
\sigma_{\alpha}(\gamma, \beta) & =\iint_{\mathbb{R}_{2}^{+}}\left(e^{i\left(\gamma x_{1}+\beta x_{2}\right)}-1\right) \phi_{\alpha}\left(d x_{1}, d x_{2}\right) \\
& =\int_{0}^{\pi / 2}\left(\int_{0}^{\infty}\left(e^{i r(\gamma \cos \theta+\beta \sin \theta)}-1\right) \frac{C \alpha}{r^{\alpha+1}} d r\right) M(d \theta) \\
& =-C \Gamma(1-\alpha) \int_{0}^{\pi / 2}[-i(\gamma \cos \theta+\beta \sin \theta)]^{\alpha} M(d \theta)
\end{aligned}
$$


where, for the last equality, we have taken into account the well-known formula

$$
\int_{0}^{\infty}\left(e^{i \xi x}-1\right) \frac{\alpha d x}{x^{\alpha+1}}=-\Gamma(1-\alpha)(-i \xi)^{\alpha} .
$$

Hence, the Laplace exponent has the form

$$
S_{\alpha}\left(\eta_{1}, \eta_{2}\right)=C \Gamma(1-\alpha) \int_{0}^{\pi / 2}\left(\eta_{1} \cos \theta+\eta_{2} \sin \theta\right)^{\alpha} M(d \theta) .
$$

Observe that $\left(\eta_{1} \cos \theta+\eta_{2} \sin \theta\right)$ is the Laplace symbol of $\left(\cos \theta \frac{\partial}{\partial x_{1}}+\sin \theta \frac{\partial}{\partial x_{2}}\right)$, which is the directional derivative along the unit vector $(\cos \theta, \sin \theta)$. Then, by taking the Laplace inverse of (2.15), the operator in (2.10) here reduces to the following pseudo-differential operator

$$
\mathcal{D}_{x_{1}, x_{2}}^{\alpha} f\left(x_{1}, x_{2}\right)=C \Gamma(1-\alpha) \int_{0}^{\pi / 2}\left(\cos \theta \frac{\partial}{\partial x_{1}}+\sin \theta \frac{\partial}{\partial x_{2}}\right)^{\alpha} M(d \theta) .
$$

Note that (2.16) represents the average under $M(d \theta)$ of the fractional power of the directional derivative along $(\cos \theta, \sin \theta)$. Thus equation (2.9) takes the form

$$
\frac{\partial}{\partial t} q_{*}\left(x_{1}, x_{2}, t\right)=-\mathcal{D}_{x_{1}, x_{2}}^{\alpha} q_{*}\left(x_{1}, x_{2}, t\right)
$$

which is a particular case of the equation governing multivariate stable processes (on this point consult [11] and [30]).

Example 2.3. Let $Y_{1}(t), Y_{2}(t)$ and $Z(t)$ be independent subordinators, and let $T_{1}(\cdot), T_{2}(\cdot)$ and $G(\cdot)$ be the respective Bernstein functions. For any $c_{1}, c_{2} \in \mathbb{R}^{+}$, we consider the bivariate subordinator

$$
\left\{\begin{array}{l}
H_{1}(t)=Y_{1}(t)+c_{1} Z(t) \\
H_{2}(t)=Y_{2}(t)+c_{2} Z(t)
\end{array}\right.
$$

Such example is motivated by the model studied in [44] and [24]; here the authors considered multivariate subordinators such that each component is a sum of an idiosyncratic and a common term, which has some interest in finance modelling. It is easy to see that (2.17) is characterized by the bivariate Bernstein function

$$
S\left(\eta_{1}, \eta_{2}\right)=T_{1}\left(\eta_{1}\right)+T_{2}\left(\eta_{2}\right)+G\left(c_{1} \eta_{1}+c_{2} \eta_{2}\right) .
$$

Then the support of the Lévy measure is the union of the coordinate axes and the line with direction $\left(c_{1}, c_{2}\right)$ passing through the origin; for the explicit expression of the Lévy measure consult Prop. 3.1 in [44].

It is interesting to note that, if $Y_{1}(t), Y_{2}(t)$ and $Z(t)$ are stable subordinators with parameter $\alpha \in(0,1)$, then the bivariate Bernstein function reads

$$
S\left(\eta_{1}, \eta_{2}\right)=\eta_{1}^{\alpha}+\eta_{2}^{\alpha}+\left(c_{1} \eta_{1}+c_{2} \eta_{2}\right)^{\alpha}
$$

thus the operator (2.10) reduces to the pseudo-differential operator

$$
\mathcal{D}_{x_{1}, x_{2}}=\frac{\partial^{\alpha}}{\partial x_{1}^{\alpha}}+\frac{\partial^{\alpha}}{\partial x_{2}^{\alpha}}+\left(c_{1} \frac{\partial}{\partial x_{1}}+c_{2} \frac{\partial}{\partial x_{2}}\right)^{\alpha}
$$

where the last summand is the fractional power of the directional derivative. 
2.2. Some results. For technical reasons that will be clear in the next section, it is sometimes convenient to consider the related bi-parameter process

$$
\left\{\left(H_{1}\left(t_{1}\right), H_{2}\left(t_{2}\right)\right), t_{1} \geq 0, t_{2} \geq 0\right\}
$$

rather than the process $\left\{\left(H_{1}(t), H_{2}(t)\right), t \geq 0\right\}$. The following result characterizes its distribution.

Proposition 2.4. The double Laplace transform of $\left(H_{1}\left(t_{1}\right), H_{2}\left(t_{2}\right)\right)$ reads

$$
\begin{aligned}
\mathbb{E} e^{-\left(\alpha H_{1}\left(t_{1}\right)+\beta H_{2}\left(t_{2}\right)\right)} & =e^{-t_{1} S(\alpha, \beta)} e^{-\left(t_{2}-t_{1}\right) T_{2}(\beta)} \mathbf{1}_{\left\{t_{2} \geq t_{1}\right\}} \\
& +e^{-t_{2} S(\alpha, \beta)} e^{-\left(t_{1}-t_{2}\right) T_{1}(\alpha)} \mathbf{1}_{\left\{t_{1}>t_{2}\right\}} \quad \alpha, \beta \geq 0,
\end{aligned}
$$

where $S, T_{1}$ and $T_{2}$ have been defined in (2.4) and (2.5), while $\mathbf{1}_{A}$ denotes the indicator function of the set $A$.

Proof. The desired equality can be checked as follows:

$$
\begin{aligned}
& \mathbb{E} e^{-\left(\alpha H_{1}\left(t_{1}\right)+\beta H_{2}\left(t_{2}\right)\right)} \\
& =\mathbb{E}\left[e^{-\left(\alpha H_{1}\left(t_{1}\right)+\beta H_{2}\left(t_{1}\right)\right)} e^{-\beta\left(H_{2}\left(t_{2}\right)-H_{2}\left(t_{1}\right)\right)}\right] \mathbf{1}_{\left\{t_{2} \geq t_{1}\right\}} \\
& +\mathbb{E}\left[e^{-\left(\alpha H_{1}\left(t_{2}\right)+\beta H_{2}\left(t_{2}\right)\right)} e^{-\alpha\left(H_{1}\left(t_{1}\right)-H_{1}\left(t_{2}\right)\right)}\right] \mathbf{1}_{\left\{t_{1}>t_{2}\right\}} \\
& =\mathbb{E}\left[e^{-\left(\alpha H_{1}\left(t_{1}\right)+\beta H_{2}\left(t_{1}\right)\right)}\right] \mathbb{E}\left[e^{-\beta\left(H_{2}\left(t_{2}\right)-H_{2}\left(t_{1}\right)\right)}\right] \mathbf{1}_{\left\{t_{2} \geq t_{1}\right\}} \\
& +\mathbb{E}\left[e^{-\left(\alpha H_{1}\left(t_{2}\right)+\beta H_{2}\left(t_{2}\right)\right)}\right] \mathbb{E}\left[e^{-\alpha\left(H_{1}\left(t_{1}\right)-H_{1}\left(t_{2}\right)\right)}\right] \mathbf{1}_{\left\{t_{1}>t_{2}\right\}} \\
& =e^{-t_{1} S(\alpha, \beta)} e^{-\left(t_{2}-t_{1}\right) T_{2}(\beta)} \mathbf{1}_{\left\{t_{2} \geq t_{1}\right\}}+e^{-t_{2} S(\alpha, \beta)} e^{-\left(t_{1}-t_{2}\right) T_{1}(\alpha)} \mathbf{1}_{\left\{t_{1}>t_{2}\right\}},
\end{aligned}
$$

where we repeatedly used independence of the increments.

From now on we assume that the random vector $\left(H_{1}(t), H_{2}(t)\right)$ has joint and marginal densities, respectively defined by

$$
\begin{aligned}
& P\left(H_{1}(t) \in d x_{1}, H_{2}(t) \in d x_{2}\right):=q_{*}\left(x_{1}, x_{2}, t\right) d x_{1} d x_{2}, \quad t>0 \\
& P\left(H_{i}(t) \in d x_{i}\right):=q_{i}\left(x_{i}, t\right) d x_{i} \quad i=1,2 \quad t>0 .
\end{aligned}
$$

Moreover

$$
P\left(H_{1}(0) \in d x_{1}, H_{2}(0) \in d x_{2}\right):=\delta_{0}\left(d x_{1}\right) \delta_{0}\left(d x_{2}\right) .
$$

Hence we introduce the joint density of $\left(H_{1}\left(t_{1}\right), H_{2}\left(t_{2}\right)\right)$ by

$$
P\left(H_{1}\left(t_{1}\right) \in d x_{1}, H_{2}\left(t_{2}\right) \in d x_{2}\right):=q\left(x_{1}, x_{2}, t_{1}, t_{2}\right) d x_{1} d x_{2} \quad \text { for all } t_{1}, t_{2}>0,
$$

where

$$
\begin{aligned}
q\left(x_{1}, x_{2}, t_{1}, t_{2}\right) & =\int_{0}^{\infty} q_{*}\left(x_{1}, z, t_{1}\right) q_{2}\left(x_{2}-z, t_{2}-t_{1}\right) d z \mathbf{1}_{\left\{t_{1} \leq t_{2}\right\}} \\
& +\int_{0}^{\infty} q_{*}\left(z, x_{2}, t_{2}\right) q_{1}\left(x_{1}-z, t_{1}-t_{2}\right) d z \mathbf{1}_{\left\{t_{1}>t_{2}\right\}},
\end{aligned}
$$

which satisfies

$$
q\left(x_{1}, x_{2}, t, t\right)=q_{*}\left(x_{1}, x_{2}, t\right) .
$$

Moreover we have that

$$
\begin{aligned}
& \lim _{t_{1} \rightarrow 0} q\left(x_{1}, x_{2}, t_{1}, t_{2}\right)=\delta_{0}\left(x_{1}\right) q_{2}\left(x_{2}, t_{2}\right) \\
& \lim _{t_{2} \rightarrow 0} q\left(x_{1}, x_{2}, t_{1}, t_{2}\right)=\delta_{0}\left(x_{2}\right) q_{1}\left(x_{1}, t_{1}\right)
\end{aligned}
$$


in the sense of distributions.

The following proposition provides a governing equation for the bi-parameter process $\left(H_{1}\left(t_{1}\right), H_{2}\left(t_{2}\right)\right)$, which involves the operator $\mathcal{D}_{x_{1}, x_{2}}$ defined in $(2.10)$. This extends (2.9) to the case where $t_{1} \neq t_{2}$.

Proposition 2.5. If $t_{1} \neq t_{2}$, then joint density $q\left(x_{1}, x_{2}, t_{1}, t_{2}\right)$ is the fundamental solution of the equation

$$
\left(\frac{\partial}{\partial t_{1}}+\frac{\partial}{\partial t_{2}}\right) q\left(x_{1}, x_{2}, t_{1}, t_{2}\right)=-\mathcal{D}_{x_{1}, x_{2}} q\left(x_{1}, x_{2}, t_{1}, t_{2}\right) \quad t_{1} \neq t_{2} \quad t_{1}, t_{2}>0
$$

under the initial conditions (2.20) and (2.21). In the case $t_{1}=t_{2}=t$, we have

$$
\frac{\partial}{\partial t} q_{*}\left(x_{1}, x_{2}, t\right)=-\mathcal{D}_{x_{1}, x_{2}} q_{*}\left(x_{1}, x_{2}, t\right) \quad t>0
$$

under the initial condition (2.19).

Proof. The Laplace transform given by Proposition 2.4 is differentiable for $t_{1} \neq t_{2}$, and, by applying the operator $\left(\frac{\partial}{\partial t_{1}}+\frac{\partial}{\partial t_{2}}\right)$ to both sides, we have

$$
\left(\frac{\partial}{\partial t_{1}}+\frac{\partial}{\partial t_{2}}\right) \mathbb{E} e^{-\left(\alpha H_{1}\left(t_{1}\right)+\beta H_{2}\left(t_{2}\right)\right)}=-S(\alpha, \beta) \mathbb{E} e^{-\left(\alpha H_{1}\left(t_{1}\right)+\beta H_{2}\left(t_{2}\right)\right)}, \quad t_{1} \neq t_{2}
$$

Then, taking into account (2.11), by the Laplace inversion of both sides we get (2.22). Finally, for $t_{1}=t_{2}=t$, by (2.3) we have

$$
\frac{\partial}{\partial t} \mathbb{E} e^{-\eta_{1} H_{1}(t)-\eta_{2} H_{2}(t)}=-S\left(\eta_{1}, \eta_{2}\right) e^{-t S\left(\eta_{1}, \eta_{2}\right)}
$$

and the inverse Laplace transform of both sides gives (2.23).

We now present a further result that will be useful later. For $t_{1}, t_{2}>0$, we define the tail of the Lévy measure $\phi$ as

$$
\bar{\phi}\left(t_{1}, t_{2}\right)=\phi\left(\left(t_{1}, \infty\right) \times\left(t_{2}, \infty\right)\right)=\int_{t_{1}}^{\infty} \int_{t_{2}}^{\infty} \phi\left(d x_{1}, d x_{2}\right) .
$$

Note that, in the case of independence, formula (2.7) yields $\bar{\phi}\left(t_{1}, t_{2}\right)=0$ for each $t_{1}$ and $t_{2}$, because $\phi$ is supported on the coordinate axes.

Proposition 2.6. The double Laplace transform of (2.25) reads

$$
\int_{0}^{\infty} \int_{0}^{\infty} e^{-\eta_{1} t_{1}-\eta_{2} t_{2}} \bar{\phi}\left(t_{1}, t_{2}\right) d t_{1} d t_{2}=\frac{T_{1}\left(\eta_{1}\right)+T_{2}\left(\eta_{2}\right)-S\left(\eta_{1}, \eta_{2}\right)}{\eta_{1} \eta_{2}},
$$

where $T_{1}, T_{2}$ and $S$ have been defined in (2.6) and (2.4).

Proof. It is sufficient to start from expression (2.4) and apply consecutively the integration by parts in both variables. The calculations exploit that

$$
\nu_{1}\left(d x_{1}\right)=\int_{0}^{\infty} \phi\left(d x_{1}, d x_{2}\right) \quad \text { and } \quad \nu_{2}\left(d x_{2}\right)=\int_{0}^{\infty} \phi\left(d x_{1}, d x_{2}\right) .
$$

The result holds by simple algebraic manipulations. 


\section{INVERSES OF BIVARIATE SUBORDINATORS}

Let $\left\{\left(H_{1}(x), H_{2}(x)\right), x \geq 0\right\}$ be a bivariate subordinator. We here define its inverse $\left\{\left(L_{1}(t), L_{2}(t)\right), t \geq 0\right\}$ as the process with marginal components

$$
L_{i}(t)=\inf \left\{x: H_{i}(x)>t\right\}, \quad i=1,2 .
$$

We also consider the related bi-parameter process $\left\{\left(L_{1}\left(t_{1}\right), L_{2}\left(t_{2}\right)\right), t_{1} \geq 0, t_{2} \geq 0\right\}$, such that

$$
L_{i}\left(t_{i}\right)=\inf \left\{x: H_{i}(x)>t_{i}\right\}, \quad i=1,2
$$

then

$$
P\left(L_{1}\left(t_{1}\right)>x_{1}, L_{2}\left(t_{2}\right)>x_{2}\right)=P\left(H_{1}\left(x_{1}\right) \leq t_{1}, H_{2}\left(x_{2}\right) \leq t_{2}\right) .
$$

It is well-known from the theory of univariate subordinators and their inverses (see, for example, [19]) that the marginal densities $l_{1}\left(x_{1}, t_{1}\right)$ and $l_{2}\left(x_{2}, t_{2}\right)$ solve the generalized fractional Cauchy problems

$$
\left\{\begin{array}{l}
\frac{\partial}{\partial x_{i}} l_{i}\left(x_{i}, t_{i}\right)=-\mathcal{D}_{t_{i}} l_{i}\left(x_{i}, t_{i}\right) \\
l_{i}\left(0, t_{i}\right)=-\mathcal{D}_{t_{i}} \theta\left(t_{i}\right)=\bar{\nu}\left(t_{i}\right) \quad i=1,2
\end{array}\right.
$$

where $\theta(\cdot)$ denotes the Heaviside function, $\mathcal{D}_{t}$ is the operator defined in (1.4), while

$$
\bar{\nu}_{k}\left(t_{k}\right)=\nu_{k}\left[t_{k}, \infty\right) \quad k=1,2
$$

is the tail of the Lévy measure. Moreover, it is well-known that

$$
\int_{0}^{\infty} e^{-\eta_{i} t_{i}} l_{i}\left(x_{i}, t_{i}\right) d t_{i}=\frac{T_{i}\left(\eta_{i}\right)}{\eta_{i}} e^{-T_{i}\left(\eta_{i}\right) x_{i}} \quad i=1,2
$$

and the space-time Laplace transforms read

$$
\int_{0}^{\infty} \int_{0}^{\infty} e^{-\eta_{i} t_{i}} e^{-\xi_{i} x_{i}} l\left(x_{i}, t_{i}\right) d x_{i} d t_{i}=\frac{T_{i}\left(\eta_{i}\right)}{\eta_{i}\left(\xi_{i}+T_{i}\left(\eta_{i}\right)\right)} \quad i=1,2 .
$$

The aim of the next subsections is to find the bi-dimensional counterparts of such results.

3.1. Distributional properties. We here assume that $\left(H_{1}\left(x_{1}\right), H_{2}\left(x_{2}\right)\right)$ is an absolutely continuous random vector with density $q\left(t_{1}, t_{2}, x_{1}, x_{2}\right)$.

In Proposition 3.1, we show that the distribution of $\left(L_{1}\left(t_{1}\right), L_{2}\left(t_{2}\right)\right)$ has two components. The first one is absolutely continuous with respect to the bi-dimensional Lebesgue measure, namely

$$
P\left(L_{1}\left(t_{1}\right) \in d x_{1}, L_{2}\left(t_{2}\right) \in d x_{2}\right)=l\left(x_{1}, x_{2}, t_{1}, t_{2}\right) d x_{1} d x_{2} \quad x_{1} \neq x_{2} ;
$$

the second one has support on the bisector line $x_{1}=x_{2}$ with one-dimensional Lebesgue density, i.e.

$$
P\left(L_{1}\left(t_{1}\right) \in d x, L_{2}\left(t_{2}\right) \in d x\right)=l_{*}\left(x, t_{1}, t_{2}\right) d x .
$$

The existence of the second component is due to the intuitive fact that the event $\left\{L_{1}\left(t_{1}\right)=L_{2}\left(t_{2}\right)\right\}$ has positive probability; in fact, since $H_{1}$ and $H_{2}$ have simultaneous jumps, the first time in which $H_{1}$ goes beyond the level $t_{1}$ may coincide with the first time in which $H_{2}$ goes beyond the level $t_{2}$.

In view of what follows, we need to introduce the Laplace transforms of $l$ and $l_{*}$ with respect to the time-variables, i.e.

$$
\tilde{l}\left(x_{1}, x_{2}, \eta_{1}, \eta_{2}\right):=\int_{0}^{\infty} \int_{0}^{\infty} e^{-\eta_{1} t_{1}-\eta_{2} t_{2}} l\left(x_{1}, x_{2}, t_{1}, t_{2}\right) d t_{1} d t_{2},
$$




$$
\tilde{l}_{*}\left(x, \eta_{1}, \eta_{2}\right):=\int_{0}^{\infty} \int_{0}^{\infty} e^{-\eta_{1} t_{1}-\eta_{2} t_{2}} l_{*}\left(x, t_{1}, t_{2}\right) d t_{1} d t_{2} .
$$

Proposition 3.1. Assume that, for each $x_{1}, x_{2}>0,\left(H_{1}\left(x_{1}\right), H_{2}\left(x_{2}\right)\right)$ has a density denoted by $q\left(t_{1}, t_{2}, x_{1}, x_{2}\right)$. Then, for any $t_{1}, t_{2}>0$, the distribution of $\left(L_{1}\left(t_{1}\right), L_{2}\left(t_{2}\right)\right)$ has an absolutely continuous component with density

$$
l\left(x_{1}, x_{2}, t_{1}, t_{2}\right)=\frac{\partial^{2}}{\partial x_{1} \partial x_{2}} \int_{0}^{t_{1}} \int_{0}^{t_{2}} q\left(\tau_{1}, \tau_{2}, x_{1}, x_{2}\right) d \tau_{1} d \tau_{2}, \quad x_{1} \neq x_{2},
$$

and its Laplace transform is

$$
\begin{aligned}
& \tilde{l}\left(x_{1}, x_{2}, \eta_{1}, \eta_{2}\right) \\
& =\frac{1}{\eta_{1} \eta_{2}} T_{2}\left(\eta_{2}\right)\left(S\left(\eta_{1}, \eta_{2}\right)-T_{2}\left(\eta_{2}\right)\right) e^{-x_{1}\left(S\left(\eta_{1}, \eta_{2}\right)-T_{2}\left(\eta_{2}\right)\right)} e^{-x_{2} T_{2}\left(\eta_{2}\right)} \mathbf{1}_{\left\{x_{1}<x_{2}\right\}} \\
& +\frac{1}{\eta_{1} \eta_{2}} T_{1}\left(\eta_{1}\right)\left(S\left(\eta_{1}, \eta_{2}\right)-T_{1}\left(\eta_{1}\right)\right) e^{-x_{2}\left(S\left(\eta_{1}, \eta_{2}\right)-T_{1}\left(\eta_{1}\right)\right)} e^{-x_{1} T_{1}\left(\eta_{1}\right)} \mathbf{1}_{\left\{x_{1}>x_{2}\right\}},
\end{aligned}
$$

where $\eta_{1}, \eta_{2} \geq 0$ and $S, T_{1}, T_{2}$ have been defined in (2.4) and (2.6). Moreover, the distribution of $\left(L_{1}\left(t_{1}\right), L_{2}\left(t_{2}\right)\right)$ also has a singular component supported on the line $x_{1}=x_{2}$, having density

$$
l_{*}\left(x, t_{1}, t_{2}\right)=\frac{\partial^{2}}{\partial x^{2}} \int_{0}^{t_{1}} \int_{0}^{t_{2}} q_{*}\left(\tau_{1}, \tau_{2}, x\right) d \tau_{1} d \tau_{2},
$$

whose Laplace transform reads

$$
\tilde{l}_{*}\left(x, \eta_{1}, \eta_{2}\right)=\frac{\left.T_{1}\left(\eta_{1}\right)+T_{2}\left(\eta_{2}\right)-S\left(\eta_{1}, \eta_{2}\right)\right)}{\eta_{1} \eta_{2}} e^{-x S\left(\eta_{1}, \eta_{2}\right)} .
$$

Proof. Since $\left(H_{1}\left(x_{1}\right), H_{2}\left(x_{2}\right)\right)$ has a density, it is possible to differentiate both sides of (3.1) and we get (3.6). For $x_{1}<x_{2}$, by using Proposition 2.4 and equation (3.6), we have

$$
\begin{aligned}
& \int_{0}^{\infty} \int_{0}^{\infty} e^{-\eta_{1} t_{1}-\eta_{2} t_{2}} l\left(x_{1}, x_{2}, t_{1}, t_{2}\right) d t_{1} d t_{2} \\
& =\int_{0}^{\infty} \int_{0}^{\infty} e^{-\eta_{1} t_{1}-\eta_{2} t_{2}} \frac{\partial^{2}}{\partial x_{1} \partial x_{2}} \int_{0}^{t_{1}} \int_{0}^{t_{2}} q\left(\tau_{1}, \tau_{2}, x_{1}, x_{2}\right) d \tau_{1} d \tau_{2} d t_{1} d t_{2} \\
& =\frac{\partial^{2}}{\partial x_{1} \partial x_{2}} \int_{0}^{\infty} \int_{0}^{\infty} q\left(\tau_{1}, \tau_{2}, x_{1}, x_{2}\right) \int_{\tau_{1}}^{\infty} \int_{\tau_{2}}^{\infty} e^{-\eta_{1} t_{1}-\eta_{2} t_{2}} d t_{1} d t_{2} d \tau_{1} d \tau_{2} \\
& =\frac{1}{\eta_{1} \eta_{2}} \frac{\partial^{2}}{\partial x_{1} \partial x_{2}} \int_{0}^{\infty} \int_{0}^{\infty} e^{-\eta_{1} \tau_{1}-\eta_{2} \tau_{2}} q\left(\tau_{1}, \tau_{2}, x_{1}, x_{2}\right) d \tau_{1} d \tau_{2} \\
& =\frac{1}{\eta_{1} \eta_{2}} \frac{\partial^{2}}{\partial x_{1} \partial x_{2}} e^{-x_{1} S\left(\eta_{1}, \eta_{2}\right)-\left(x_{2}-x_{1}\right) T_{2}\left(\eta_{2}\right)} \\
& =\frac{1}{\eta_{1} \eta_{2}} T_{2}\left(\eta_{2}\right)\left(S\left(\eta_{1}, \eta_{2}\right)-T_{2}\left(\eta_{2}\right)\right) e^{-x_{1}\left(S\left(\eta_{1}, \eta_{2}\right)-T_{2}\left(\eta_{2}\right)\right)} e^{-x_{2} T_{2}\left(\eta_{2}\right)} .
\end{aligned}
$$

The result for the case $x_{1}>x_{2}$ can be proved similarly. We also remark that

$$
\int_{0}^{\infty} \int_{0}^{\infty} l\left(x_{1}, x_{2}, t_{1}, t_{2}\right) d x_{1} d x_{2} \neq 1
$$

as

$$
\int_{0}^{\infty} \int_{0}^{\infty} e^{-\eta_{1} t_{1}-\eta_{2} t_{2}}\left(\int_{0}^{\infty} \int_{0}^{\infty} l\left(x_{1}, x_{2}, t_{1}, t_{2}\right) d x_{1} d x_{2}\right) d t_{1} d t_{2} \neq \frac{1}{\eta_{1} \eta_{2}} .
$$


This proves that $P\left(L_{1}\left(t_{1}\right)=L_{2}\left(t_{2}\right)\right)>0$. By putting $x_{1}=x_{2}=x$ in (3.1), and taking the derivatives with respect to $x$ of both sides, equation (3.8) is obtained. To compute (3.9) we can use the following representation

$$
l_{*}\left(x, t_{1}, t_{2}\right)=\int_{0}^{t_{1}} \int_{0}^{t_{2}} \bar{\phi}\left(t_{1}-s_{1}, t_{2}-s_{2}\right) q_{*}\left(s_{1}, s_{2}, x\right) d s_{1} d s_{2}
$$

and apply the convolution theorem for the Laplace transforms, by considering expressions (2.26) and (2.3).

Remark 3.2. Of course, the following normalizing condition holds:

$$
\int_{0}^{\infty} \int_{0}^{\infty} l\left(x_{1}, x_{2}, t_{1}, t_{2}\right) d x_{1} d x_{2}+\int_{0}^{\infty} l_{*}\left(x, t_{1}, t_{2}\right) d x=1 .
$$

In fact we can check (3.10) by using the Laplace transforms in Proposition 3.1, and we get

$$
\begin{aligned}
& \int_{0}^{\infty} \int_{0}^{\infty} e^{-\eta_{1} t_{1}-\eta_{2} t_{2}}\left(\int_{0}^{\infty} \int_{0}^{\infty} l\left(x_{1}, x_{2}, t_{1}, t_{2}\right) d x_{1} d x_{2}+\int_{0}^{\infty} l_{*}\left(x, t_{1}, t_{2}\right) d x\right) d t_{1} d t_{2} \\
& =\frac{1}{\eta_{1} \eta_{2}} .
\end{aligned}
$$

Remark 3.3. Proposition 3.1 gives the time-Laplace transform of the distribution, presenting three different expressions related to the regions $x_{1}\left\langle x_{2}, x_{1}\right\rangle x_{2}$ and $x_{1}=x_{2}$. These three components are jointly taken into account in the following space Laplace transform:

$$
\begin{aligned}
& \int_{0}^{\infty} \int_{0}^{\infty} \int_{0}^{\infty} \int_{0}^{\infty} e^{-\eta_{1} t_{1}-\eta_{2} t_{2}} e^{-\xi_{1} x_{1}-\xi_{2} x_{2}} P\left(L_{1}\left(t_{1}\right) \in d x_{1}, L_{2}\left(t_{2}\right) \in d x_{2}\right) d t_{1} d t_{2} \\
& =\int_{0}^{\infty} \int_{0}^{\infty} e^{-\xi_{1} x_{1}-\xi_{2} x_{2}} \tilde{l}\left(x_{1}, x_{2}, \eta_{1}, \eta_{2}\right) d x_{1} d x_{2}+\int_{0}^{\infty} e^{-\left(\xi_{1}+\xi_{2}\right) x} \tilde{l}_{*}\left(x, \eta_{1}, \eta_{2}\right) d x \\
& =\frac{T_{1}\left(\eta_{1}\right) T_{2}\left(\eta_{2}\right)}{\eta_{1} \eta_{2}\left[\xi_{1}+T_{1}\left(\eta_{1}\right)\right]\left[\xi_{2}+T_{2}\left(\eta_{2}\right)\right]}+\frac{\xi_{1} \xi_{2}\left[T_{1}\left(\eta_{1}\right)+T_{2}\left(\eta_{2}\right)-S\left(\eta_{1}, \eta_{1}\right)\right]}{\eta_{1} \eta_{2}\left[\xi_{1}+T_{1}\left(\eta_{1}\right)\right]\left[\xi_{2}+T_{2}\left(\eta_{2}\right)\right]\left[\xi_{1}+\xi_{2}+S\left(\eta_{1}, \eta_{1}\right)\right]}
\end{aligned}
$$

for $\xi_{1}, \xi_{2}, \eta_{1}, \eta_{2}>0$. We remark that, in the case of independence, by using (2.8) we have that (3.11) reduces to the product of the univariate transforms given in (3.3).

In the following proposition we prove that the survival function of $\left(L_{1}\left(t_{1}\right), L_{2}\left(t_{2}\right)\right)$ decays at least exponentially fast. Therefore all the mixed moments of integer order exist. We then compute the Laplace transform for the covariance between $L_{1}\left(t_{1}\right)$ and $L_{2}\left(t_{2}\right)$.

Proposition 3.4. For any $\eta_{1}, \eta_{2} \geq 0$ the following inequality holds

$$
\begin{aligned}
P\left(L_{1}\left(t_{1}\right) \geq x_{1}, L_{2}\left(t_{2}\right) \geq x_{2}\right) \leq & e^{-x_{1} S\left(\eta_{1}, \eta_{2}\right)} e^{-\left(x_{2}-x_{1}\right) T_{2}\left(\eta_{2}\right)} e^{\eta_{1} t_{1}} e^{\eta_{2} t_{2}} \mathbf{1}_{\left\{x_{2} \geq x_{1}\right\}} \\
& +e^{-x_{2} S\left(\eta_{1}, \eta_{2}\right)} e^{-\left(x_{1}-x_{2}\right) T_{1}\left(\eta_{1}\right)} e^{\eta_{1} t_{1}} e^{\eta_{2} t_{2}} \mathbf{1}_{\left\{x_{1}>x_{2}\right\}} .
\end{aligned}
$$

Therefore, all the mixed moments of integer order exist and, moreover,

$$
\int_{0}^{\infty} \int_{0}^{\infty} e^{-\eta_{1} t_{1}-\eta_{2} t_{2}} \operatorname{cov}\left(L_{1}\left(t_{1}\right), L_{2}\left(t_{2}\right)\right) d t_{1} d t_{2}=\frac{T_{1}\left(\eta_{1}\right)+T_{2}\left(\eta_{2}\right)-S\left(\eta_{1}, \eta_{2}\right)}{\eta_{1} \eta_{2} T_{1}\left(\eta_{1}\right) T_{2}\left(\eta_{2}\right) S\left(\eta_{1}, \eta_{2}\right)} .
$$


Proof. The proof of (3.12) is based on the so-called bivariate Markov inequality (see [38], Theorem 1). For $\eta_{1}, \eta_{2} \geq 0$,

$$
\begin{aligned}
P\left(L_{1}\left(t_{1}\right) \geq x_{1}, L_{2}\left(t_{2}\right) \geq x_{2}\right) & =P\left(H_{1}\left(x_{1}\right)<t_{1}, H_{2}\left(x_{2}\right)<t_{2}\right) \\
& =P\left(e^{-\eta_{1} H_{1}\left(x_{1}\right)}>e^{-\eta_{1} t_{1}}, e^{-\eta_{2} H_{2}\left(x_{2}\right)}>e^{-\eta_{2} t_{2}}\right) \\
& \leq \frac{\mathbb{E}\left[e^{-\eta_{1} H_{1}\left(x_{1}\right)-\eta_{2} H_{2}\left(x_{2}\right)}\right]}{e^{-\eta_{1} t_{1}} e^{-\eta_{2} t_{2}}} \\
& =e^{-x_{1} S\left(\eta_{1}, \eta_{2}\right)} e^{-\left(x_{2}-x_{1}\right) T_{2}\left(\eta_{2}\right)} e^{\eta_{1} t_{1}} e^{\eta_{2} t_{2}} \mathbf{1}_{\left\{x_{2} \geq x_{1}\right\}} \\
& +e^{-x_{2} S\left(\eta_{1}, \eta_{2}\right)} e^{-\left(x_{1}-x_{2}\right) T_{1}\left(\eta_{1}\right)} e^{\eta_{1} t_{1}} e^{\eta_{2} t_{2}} \mathbf{1}_{\left\{x_{1}>x_{2}\right\}}
\end{aligned}
$$

where the last equality holds by Proposition 2.4. The Laplace transform of $\mathbb{E}\left(L_{1}\left(t_{1}\right) L_{2}\left(t_{2}\right)\right)$ can be computed as

$$
\begin{aligned}
& \int_{0}^{\infty} \int_{0}^{\infty} e^{-\eta_{1} t_{1}-\eta_{2} t_{2}} \mathbb{E}\left(L_{1}\left(t_{1}\right) L_{2}\left(t_{2}\right)\right) d t_{1} d t_{2} \\
& =\int_{0}^{\infty} \int_{0}^{\infty} x_{1} x_{2} \tilde{l}\left(x_{1}, x_{2}, \eta_{1}, \eta_{2}\right) d x_{1} d x_{2}+\int_{0}^{\infty} x^{2} \tilde{l}_{*}\left(x, \eta_{1}, \eta_{2}\right) d x
\end{aligned}
$$

and then, exploiting Prop. 3.1, after some calculations we get

$$
\int_{0}^{\infty} \int_{0}^{\infty} e^{-\eta_{1} t_{1}-\eta_{2} t_{2}} \mathbb{E}\left(L_{1}\left(t_{1}\right) L_{2}\left(t_{2}\right)\right) d t_{1} d t_{2}=\frac{T_{1}\left(\eta_{1}\right)+T_{2}\left(\eta_{2}\right)}{\eta_{1} \eta_{2} T_{1}\left(\eta_{1}\right) T_{2}\left(\eta_{2}\right) S\left(\eta_{1}, \eta_{2}\right)} .
$$

Finally, by taking into account that univariate inverse subordinators are such that

$$
\int_{0}^{\infty} e^{-\eta_{i} t_{i}} \mathbb{E} L_{i}\left(t_{i}\right) d t_{i}=\frac{1}{\eta_{i} T_{i}\left(\eta_{i}\right)} \quad i=1,2,
$$

we immediately obtain (3.13).

Remark 3.5. It is known that, if $H$ is a univariate subordinator and $L$ is its inverse, then $\mathbb{E} L(t)$ can be related to the potential density of $H$. We recall that, in the frame of potential theory (see e.g. [9]), the potential density of a subordinator $H$ is the function $t \rightarrow u(t)$ such that $u(t) d t$ represents the mean sojourn time spent by $H$ in the interval $[t, t+d t)$, namely

$$
u(t) d t=\int_{0}^{\infty} P(H(x) \in d t) d x
$$

indeed the following relation holds

$$
u(t)=\frac{d}{d t} \mathbb{E} L(t)
$$

It is interesting to remark that a similar result holds for a bivariate subordinator $\left(H_{1}, H_{2}\right)$ with inverse $\left(L_{1}, L_{2}\right)$. Indeed, let $u\left(t_{1}, t_{2}\right) d t_{1} d t_{2}$ be the mean sojourn time spent by $\left(H_{1}, H_{2}\right)$ in the set $\left[t_{1}, t_{1}+d t_{1}\right) \times\left[t_{2}, t_{2}+d t_{2}\right)$, i.e.

$$
u\left(t_{1}, t_{2}\right) d t_{1} d t_{2}=\iint_{\mathbb{R}_{+}^{2}} P\left(H_{1}\left(x_{1}\right) \in d t_{1}, H_{2}\left(x_{2}\right) \in d t_{2}\right) d x_{1} d x_{2} ;
$$

then the following relation holds

$$
\begin{aligned}
\frac{\partial^{2}}{\partial t_{1} \partial t_{2}} \mathbb{E}\left(L_{1}\left(t_{1}\right) L_{2}\left(t_{2}\right)\right) & =\frac{\partial^{2}}{\partial t_{1} \partial t_{2}} \iint_{\mathbb{R}_{+}^{2}} P\left(L_{1}\left(t_{1}\right)>x_{1}, L_{2}\left(t_{2}\right)>x_{2}\right) d x_{1} d x_{2} \\
& =\frac{\partial^{2}}{\partial t_{1} \partial t_{2}} \iint_{\mathbb{R}_{+}^{2}} P\left(H_{1}\left(x_{1}\right) \leq t_{1}, H_{2}\left(x_{2}\right) \leq t_{2}\right) d x_{1} d x_{2}
\end{aligned}
$$




$$
=u\left(t_{1}, t_{2}\right) .
$$

3.2. Governing equation. The following theorem provides boundary value problems for both the densities $l\left(x_{1}, x_{2}, t_{1}, t_{2}\right)$ and $l_{*}\left(x, t_{1}, t_{2}\right)$ defined in (3.4) and (3.5). In this way we generalize the boundary value problems in (3.2). The arising equations are PDEs exhibiting ordinary derivatives in the space variables and the integro-differential operator $\mathcal{D}_{t_{1}, t_{2}}$ in the time variables. We note that, for wellposedness reasons, we set two distinct boundary value problems for $l$, which are respectively defined on the open sets $x_{2}>x_{1}>0$ and $x_{1}>x_{2}>0$. We will denote again the Heaviside function by $\theta(\cdot)$.

Theorem 3.6. The density $l\left(x_{1}, x_{2}, t_{1}, t_{2}\right)$ solves the equation

$$
\left(\frac{\partial}{\partial x_{1}}+\frac{\partial}{\partial x_{2}}\right) l\left(x_{1}, x_{2}, t_{1}, t_{2}\right)=-\mathcal{D}_{t_{1}, t_{2}} l\left(x_{1}, x_{2}, t_{1}, t_{2}\right)
$$

on the open set $x_{2}>x_{1}>0$, under the boundary condition

$$
l\left(0, x_{2}, t_{1}, t_{2}\right)=\left(\mathcal{D}_{t_{1}, t_{2}}-\mathcal{D}_{t_{2}}^{(2)}\right) \mathcal{D}_{t_{2}}^{(2)} \theta\left(t_{1}\right) P\left(L_{2}\left(t_{2}\right) \geq x_{2}\right), \quad x_{2}>0,
$$

where $\mathcal{D}_{x}^{(i)}$ has been defined in (2.12). Moreover, on the open set $x_{1}>x_{2}>0$, $l\left(x_{1}, x_{2}, t_{1}, t_{2}\right)$ solves the same equation (3.15) under the boundary condition

$$
l\left(x_{1}, 0, t_{1}, t_{2}\right)=\left(\mathcal{D}_{t_{1}, t_{2}}-\mathcal{D}_{t_{1}}^{(1)}\right) \mathcal{D}_{t_{1}}^{(1)} \theta\left(t_{2}\right) P\left(L_{1}\left(t_{1}\right) \geq x_{1}\right) \quad x_{1}>0 .
$$

Furthermore, the density $l_{*}\left(x, t_{1}, t_{2}\right)$ solves the equation

$$
\frac{\partial}{\partial x} l_{*}\left(x, t_{1}, t_{2}\right)=-\mathcal{D}_{t_{1}, t_{2}} l_{*}\left(x, t_{1}, t_{2}\right) \quad x>0
$$

under the boundary condition

$$
l_{*}\left(0, t_{1}, t_{2}\right)=\bar{\phi}\left(t_{1}, t_{2}\right)
$$

where $\bar{\phi}$ has been defined in (2.25).

Proof. We start with the case $x_{2}>x_{1}>0$; the case $x_{1}>x_{2}>0$ can be treated similarly. We apply $\frac{\partial}{\partial x_{1}}+\frac{\partial}{\partial x_{2}}$ to both members of (3.6); by using Proposition 2.5 we have

$$
\left(\frac{\partial}{\partial x_{1}}+\frac{\partial}{\partial x_{2}}\right) l\left(x_{1}, x_{2}, t_{1}, t_{2}\right)=-\frac{\partial^{2}}{\partial x_{1} \partial x_{2}} \int_{0}^{t_{1}} \int_{0}^{t_{2}} \mathcal{D}_{\tau_{1}, \tau_{2}} q\left(\tau_{1}, \tau_{2}, x_{1}, x_{2}\right) d \tau_{1} d \tau_{2} .
$$

By using the definition of $\mathcal{D}_{\tau_{1}, \tau_{2}}$, Fubini's theorem gives

$$
\int_{0}^{t_{1}} \int_{0}^{t_{2}} \mathcal{D}_{\tau_{1}, \tau_{2}} q\left(\tau_{1}, \tau_{2}, x_{1}, x_{2}\right) d \tau_{1} d \tau_{2}=\mathcal{D}_{t_{1}, t_{2}} \int_{0}^{t_{1}} \int_{0}^{t_{2}} q\left(\tau_{1}, \tau_{2}, x_{1}, x_{2}\right) d \tau_{1} d \tau_{2}
$$

which leads to equation (3.15). In order to check the boundary condition (3.16), we put $x_{1}=0$ in (3.7), which yields

$$
\tilde{l}\left(0, x_{2}, \eta_{1}, \eta_{2}\right)=\frac{1}{\eta_{1} \eta_{2}} T_{2}\left(\eta_{2}\right)\left(S\left(\eta_{1}, \eta_{2}\right)-T_{2}\left(\eta_{2}\right)\right) e^{-x_{2} T_{2}\left(\eta_{2}\right)}
$$

and the inversion of the Laplace transform gives (3.16).

We finally conclude with the results for the density $l_{*}\left(x, t_{1}, t_{2}\right)$. In order to obtain equation (3.18) we have to apply $\frac{\partial}{\partial x}$ to both sides of (3.8) and use (2.9) together 
with the fact that $\mathcal{D}_{t_{1}, t_{2}}$ commutes with the integral. Finally, by putting $x=0$ in (3.9) we get

$$
\tilde{l}_{*}\left(0, \eta_{1}, \eta_{2}\right)=\frac{T_{1}\left(\eta_{1}\right)+T_{2}\left(\eta_{2}\right)-S\left(\eta_{1}, \eta_{2}\right)}{\eta_{1} \eta_{2}}
$$

by taking into account (2.26), the inverse Laplace transform gives the boundary condition (3.19).

Remark 3.7. The boundary condition (3.19) means that, heuristically speaking, $H_{1}$ and $H_{2}$ perform initial jumps respectively greater than $t_{1}$ and $t_{2}$ if and only if the first hitting times of the levels $t_{1}$ and $t_{2}$ are both equal to 0.

Remark 3.8. We here discuss the well-posedness of the boundary value problems in Thm. 3.6. For the region $x_{2}>x_{1}>0$, by applying the time-Laplace transform to (3.15), one has

$$
\left(\frac{\partial}{\partial x_{1}}+\frac{\partial}{\partial x_{2}}\right) \tilde{l}\left(x_{1}, x_{2}, \eta_{1}, \eta_{2}\right)=-S\left(\eta_{1}, \eta_{2}\right) \tilde{l}\left(x_{1}, x_{2}, \eta_{1}, \eta_{2}\right)
$$

which is a damped wave equation in the variables $x_{1}$ and $x_{2}$, under the boundary condition

$$
\tilde{l}\left(0, x_{2}, \eta_{1}, \eta_{2}\right)=\frac{1}{\eta_{1} \eta_{2}} T_{2}\left(\eta_{2}\right)\left(S\left(\eta_{1}, \eta_{2}\right)-T_{2}\left(\eta_{2}\right) e^{-x_{2} T_{2}\left(\eta_{2}\right)} .\right.
$$

Such a problem can be solved by the method of characteristics which gives the desired solution (3.7). Indeed the characteristic lines are $x_{2}=x_{1}+k$, with $k>0$ which carry the boundary condition to the region $x_{2}>x_{1}>0$, giving the solution

$$
\tilde{l}\left(x_{1}, x_{2}, \eta_{1}, \eta_{2}\right)=\tilde{l}\left(0, x_{2}-x_{1}, \eta_{1}, \eta_{2}\right) e^{-S\left(\eta_{1}, \eta_{2}\right) x_{1}}
$$

which coincides with (3.7). In the same way, the characteristic lines $x_{2}=x_{1}+k$, with $k<0$, carry the boundary condition $\tilde{l}\left(x_{1}, 0, \eta_{1}, \eta_{2}\right)$ to the region $x_{1}>x_{2}>0$ and lead to the solution.

Finally, by passing again to Laplace transform in the time variables, it is straightforward to check the well-posedness of the boundary value problem for $l_{*}\left(x, t_{1}, t_{2}\right)$, as $\tilde{l}_{*}\left(x, \eta_{1}, \eta_{2}\right)$ is governed by an ordinary differential equation in the variable $x$.

\section{Time-Change of Bivariate Markov processes}

Let $\left(X_{1}(t), X_{2}(t)\right)$ be a $\mathbb{R}^{2}$-valued Markov process such that $X_{1}(t)$ and $X_{2}(t)$ are independent. Without loss of generality, we assume that $\left(X_{1}(0), X_{2}(0)\right)=(0,0)$ almost surely. Let $p_{i}\left(x_{i}, t\right), i=1,2$, be the marginal density of $X_{i}(t)$, and let $p_{*}\left(x_{1}, x_{2}, t\right)=p_{1}\left(x_{1}, t\right) p_{2}\left(x_{2}, t\right)$ denote the joint density. The following forward equations hold:

$$
\begin{aligned}
\frac{\partial}{\partial t} p_{i}\left(x_{i}, t\right) & =\mathcal{G}_{i} p_{i}\left(x_{i}, t\right) \quad i=1,2 \\
\frac{\partial}{\partial t} p_{*}\left(x_{1}, x_{2}, t\right) & =\left(\mathcal{G}_{1}+\mathcal{G}_{2}\right) p_{*}\left(x_{1}, x_{2}, t\right),
\end{aligned}
$$

where the operators $\mathcal{G}_{1}$ and $\mathcal{G}_{2}$ are the duals to the generators of $X_{1}$ and $X_{2}$. It is straightforward to verify that the bi-parameter process $\left(X_{1}\left(t_{1}\right), X_{2}\left(t_{2}\right)\right)$ has a density $p\left(x_{1}, x_{2}, t_{1}, t_{2}\right)=p_{1}\left(x_{1}, t_{1}\right) p_{2}\left(x_{2}, t_{2}\right)$ satisfying

$$
\left(\frac{\partial}{\partial t_{1}}+\frac{\partial}{\partial t_{2}}\right) p\left(x_{1}, x_{2}, t_{1}, t_{2}\right)=\left(\mathcal{G}_{1}+\mathcal{G}_{2}\right) p\left(x_{1}, x_{2}, t_{1}, t_{2}\right) .
$$


We are now interested in the time-changed process

$$
\left\{\left(X_{1}\left(L_{1}\left(t_{1}\right)\right), X_{2}\left(L_{2}\left(t_{2}\right)\right)\right), t_{1} \geq 0, t_{2} \geq 0\right\},
$$

where $\left(L_{1}, L_{2}\right)$ is the inverse of a bivariate subordinator, which is independent on $\left(X_{1}, X_{2}\right)$. By a simple conditioning argument, the density of the random vector $\left(X_{1}\left(L_{1}\left(t_{1}\right)\right), X_{2}\left(L_{2}\left(t_{2}\right)\right)\right)$ has the form

$$
\begin{aligned}
h\left(x_{1}, x_{2}, t_{1}, t_{2}\right)= & \int_{0}^{\infty} \int_{0}^{\infty} p\left(x_{1}, x_{2}, u, v\right) l\left(u, v, t_{1}, t_{2}\right) d u d v \\
& +\int_{0}^{\infty} p_{*}\left(x_{1}, x_{2}, u\right) l_{*}\left(u, t_{1}, t_{2}\right) d u
\end{aligned}
$$

where the functions $l$ and $l_{*}$ have been defined in (3.4) and (3.5). The following theorem provides a governing equation for (4.5), which turns out to be a generalization of eq. (4.3) as the operator $\frac{\partial}{\partial t_{1}}+\frac{\partial}{\partial t_{2}}$ on the left hand side is replaced by $\mathcal{D}_{t_{1}, t_{2}}$ defined in (2.10).

Theorem 4.1. Let $\left(X_{1}(t), X_{2}(t)\right)$ be a $\mathbb{R}^{2}$-valued Markov process, with independent marginal components, such that $\left(X_{1}(0), X_{2}(0)\right)=(0,0)$ almost surely. Moreover, let (4.1) and (4.2) hold. Then, for any $t_{1}, t_{2}>0$, the density (4.5) of the time-changed process $\left(X_{1}\left(L_{1}\left(t_{1}\right)\right), X_{2}\left(L_{2}\left(t_{2}\right)\right)\right.$ satisfies the following equation

$$
\mathcal{D}_{t_{1}, t_{2}} h\left(x_{1}, x_{2}, t_{1}, t_{2}\right)=\left(\mathcal{G}_{1}+\mathcal{G}_{2}\right) h\left(x_{1}, x_{2}, t_{1}, t_{2}\right) \quad x_{1} \neq 0, x_{2} \neq 0 .
$$

Proof. By applying $\mathcal{D}_{t_{1}, t_{2}}$ to both sides of (4.5) and using Thm. 3.6 we have

$$
\begin{aligned}
& \mathcal{D}_{t_{1}, t_{2}} h\left(x_{1}, x_{2}, t_{1}, t_{2}\right) \\
& =-\int_{0}^{\infty} \int_{0}^{\infty} p\left(x_{1}, x_{2}, u, v\right) \frac{\partial}{\partial u} l\left(u, v, t_{1}, t_{2}\right) d u d v \\
& -\int_{0}^{\infty} \int_{0}^{\infty} p\left(x_{1}, x_{2}, u, v\right) \frac{\partial}{\partial v} l\left(u, v, t_{1}, t_{2}\right) d u d v \\
& -\int_{0}^{\infty} p_{*}\left(x_{1}, x_{2}, u\right) \frac{\partial}{\partial u} l_{*}\left(u, t_{1}, t_{2}\right) d u .
\end{aligned}
$$

Now, we integrate by parts and use (4.1) and (4.2), together with the fact that $\left(X_{1}(0), X_{2}(0)\right)=(0,0)$ almost surely; thus we get

$$
\begin{aligned}
& \mathcal{D}_{t_{1}, t_{2}} h\left(x_{1}, x_{2}, t_{1}, t_{2}\right) \\
& =\mathcal{G}_{1} \int_{0}^{\infty} \int_{0}^{\infty} p\left(x_{1}, x_{2}, u, v\right) l\left(u, v, t_{1}, t_{2}\right) d u d v+\delta\left(x_{1}\right) \int_{0}^{\infty} p_{2}\left(x_{2}, v\right) l\left(0, v, t_{1} t_{2}\right) d v+ \\
& +\mathcal{G}_{2} \int_{0}^{\infty} \int_{0}^{\infty} p\left(x_{1}, x_{2}, u, v\right) l\left(u, v, t_{1}, t_{2}\right) d u d v+\delta\left(x_{2}\right) \int_{0}^{\infty} p_{1}\left(x_{1}, u\right) l\left(u, 0, t_{1} t_{2}\right) d u \\
& +\left(\mathcal{G}_{1}+\mathcal{G}_{2}\right) \int_{0}^{\infty} p_{*}\left(x_{1}, x_{2}, u\right) l_{*}\left(u, t_{1}, t_{2}\right) d u+\delta\left(x_{1}\right) \delta\left(x_{2}\right) \bar{\phi}\left(t_{1}, t_{2}\right)
\end{aligned}
$$

where $\bar{\phi}\left(t_{1}, t_{2}\right)$ has been defined in (2.25). In the region $x_{1} \neq 0, x_{2} \neq 0$ we have

$$
\begin{aligned}
& \mathcal{D}_{t_{1}, t_{2}} h\left(x_{1}, x_{2}, t_{1}, t_{2}\right) \\
= & \left(\mathcal{G}_{1}+\mathcal{G}_{2}\right) \int_{0}^{\infty} \int_{0}^{\infty} p\left(x_{1}, x_{2}, u, v\right) l\left(u, v, t_{1}, t_{2}\right) d u d v \\
& +\left(\mathcal{G}_{1}+\mathcal{G}_{2}\right) \int_{0}^{\infty} p_{*}\left(x_{1}, x_{2}, u\right) l_{*}\left(u, t_{1}, t_{2}\right) d u
\end{aligned}
$$




$$
=\left(\mathcal{G}_{1}+\mathcal{G}_{2}\right) h\left(x_{1}, x_{2}, t_{1}, t_{2}\right),
$$

which concludes the proof.

4.1. Time-change of continuous-time Markov chains. We specialize the framework of this section by considering the case where $X_{1}(t)$ and $X_{2}(t)$ are independent continuous-time Markov chains such that $\left(X_{1}(0), X_{2}(0)\right)=(0,0)$ almost surely. In particular, they are defined as

$$
X_{l}(t)=X_{l}^{(n)} \quad T_{l}^{(n)} \leq t<T_{l}^{(n+1)} \quad l=1,2,
$$

where $X_{1}^{(n)}$ and $X_{2}^{(n)}, n \in \mathbb{N}_{0}$, denote the embedded Markov chains, with values on the discrete spaces $\mathcal{S}_{1} \subset \mathbb{R}$ and $\mathcal{S}_{2} \subset \mathbb{R}$ respectively, while all the inter-arrival times $T_{l}^{(n+1)}-T_{l}^{(n)}$ have exponential distribution with mean $1 / \xi_{l}$. The transition matrices of the embedded chains are

$$
P\left(X_{1}^{(n+1)}=j \mid X_{1}^{(n)}=i\right):=A_{i j}, \quad i, j \in \mathcal{S}_{1}
$$

and

$$
P\left(X_{2}^{(n+1)}=j \mid X_{2}^{(n)}=i\right):=B_{i j}, \quad i, j \in \mathcal{S}_{2} .
$$

Let $\left(L_{1}(t), L_{2}(t)\right)$ be again the inverse of a bivariate subordinator. We are interested in the time-changed process $\left(X_{1}\left(L_{1}(t)\right), X_{2}\left(L_{2}(t)\right)\right)$, with values in $\mathcal{S}_{1} \times \mathcal{S}_{2}$, which is defined by

$$
X_{l}\left(L_{l}(t)\right)=X_{l}^{(n)} \quad T_{l}^{(n)} \leq L_{l}(t)<T_{l}^{(n+1)}, \quad l=1,2,
$$

or, equivalently, by

$$
X_{l}\left(L_{l}(t)\right)=X_{l}^{(n)} \quad H_{l}\left(T_{l}^{(n)}\right) \leq t<H_{l}\left(T_{l}^{(n+1)}\right), \quad l=1,2 .
$$

We remark that, by Theorem 4.1, the density $p\left(x_{1}, x_{2}, t_{1}, t_{2}\right)$ of the random vector $\left(X_{1}\left(L_{1}\left(t_{1}\right)\right), X_{2}\left(L_{2}\left(t_{2}\right)\right)\right)$ solves the following equation for $x_{1}, x_{2} \neq 0$ :

$$
\begin{aligned}
\mathcal{D}_{t_{1} t_{2}} p\left(x_{1}, x_{2}, t_{1}, t_{2}\right) & =\xi_{1} \sum_{k \in S_{1}}\left(A_{k, x_{1}}-\delta_{k, x_{1}}\right) p\left(k, x_{2}, t_{1}, t_{2}\right) \\
& +\xi_{2} \sum_{k \in S_{2}}\left(B_{k, x_{2}}-\delta_{k, x_{2}}\right) p\left(x_{1}, k, t_{1}, t_{2}\right),
\end{aligned}
$$

where the matrices $A_{i j}$ and $B_{i j}$ have been defined in (4.9) and (4.10).

Clearly, for each $l=1,2$, we have that $X_{l}\left(L_{l}(t)\right)$ is a stepped semi-Markov process with inter-arrival times $\Delta_{l, n}=H_{l}\left(T_{l}^{(n+1)}\right)-H_{l}\left(T_{l}^{(n)}\right)$. This class of processes has been widely studied in the literature concerning the classical time-change by inverse subordinators (see, in particular [36] and [42] for the subordination of continuous time Markov chains). Since $H_{l}$ has stationary increments, the random variables $\Delta_{l, n}$, are i.i.d. copies of $H_{l}\left(W_{l}\right)$, where $W_{l}$ is exponentially distributed with mean $1 / \xi_{l}$.

Remark 4.2. It is important to note that the time-change by $\left(L_{1}, L_{2}\right)$ introduces dependence between the marginal components. Indeed, on the one hand, the Markov processes $X_{1}(t)$ and $X_{2}(t)$ are respectively characterized by the inter-arrival times following the laws of the random variables $W_{1}$ and $W_{2}$ cited above; on the other hand, the processes $X_{1}\left(L_{1}(t)\right)$ and $X_{2}\left(L_{2}(t)\right)$ exhibit new inter-arrival times respectively distribuited as $H_{1}\left(W_{1}\right)$ and $H_{2}\left(W_{2}\right)$ (which are dependent because of the dependence between $\mathrm{H}_{1}$ and $\mathrm{H}_{2}$ ). 
The survival functions of $H_{i}\left(W_{i}\right), i=1,2$ satisfy the following relation

$$
P\left(H_{i}\left(W_{i}\right)>t\right)=\mathbb{E} e^{-\xi_{i} L_{i}(t)} .
$$

For example, in the special case where $L_{i}(t)$ is the inverse of a stable subordinator with index $\alpha \in(0,1)$, the following explicit expression holds

$$
P\left(H_{i}\left(W_{i}\right)>t\right)=\mathcal{E}_{\alpha}\left(-\xi_{i} t^{\alpha}\right)
$$

where $\mathcal{E}_{\alpha}(x)=\sum_{k=0}^{\infty} \frac{x^{k}}{\Gamma(1+\alpha k)}$ is the one-parameter Mittag-Leffler function.

It is interesting to note that the couple of dependent inter-arrival times $\left(H_{1}\left(W_{1}\right), H_{2}\left(W_{2}\right)\right)$ has joint distribution such that

$$
P\left(H_{1}\left(W_{1}\right)>t_{1}, H_{2}\left(W_{2}\right)>t_{2}\right)=\mathbb{E} e^{-\xi_{1} L_{1}\left(t_{1}\right)-\xi_{2} L_{2}\left(t_{2}\right)},
$$

which clearly generalizes (4.12). The proof is straightforward:

$$
\begin{aligned}
& P\left(H_{1}\left(W_{1}\right)>t_{1}, H_{2}\left(W_{2}\right)>t_{2}\right) \\
& =\xi_{1} \xi_{2} \int_{0}^{\infty} \int_{0}^{\infty} P\left(H_{1}\left(w_{1}\right)>t_{1}, H_{2}\left(w_{2}\right)>t_{2}\right) e^{-\xi_{1} w_{1}} e^{-\xi_{2} w_{2}} d w_{1} d w_{2} \\
& =\xi_{1} \xi_{2} \int_{0}^{\infty} \int_{0}^{\infty} P\left(L_{1}\left(t_{1}\right)<w_{1}, L_{2}\left(t_{2}\right)<w_{2}\right) e^{-\xi_{1} w_{1}} e^{-\xi_{2} w_{2}} d w_{1} d w_{2} \\
& =\int_{0}^{\infty} \int_{0}^{\infty} P\left(L_{1}\left(t_{1}\right) \in d w_{1}, L_{2}\left(t_{2}\right) \in d w_{2}\right) e^{-\xi_{1} w_{1}} e^{-\xi_{2} w_{2}} \\
& =\mathbb{E} e^{-\xi_{1} L_{1}\left(t_{1}\right)-\xi_{2} L_{2}\left(t_{2}\right)} .
\end{aligned}
$$

4.1.1. Bivariate fractional Poisson processes. Let us consider the special case where $X_{1}(t)$ and $X_{2}(t)$ are Poisson processes $N_{1}(t)$ and $N_{2}(t)$ with intensities $\xi_{1}$ and $\xi_{2}$, while $\left(L_{1}^{\alpha}(t), L_{2}^{\alpha}(t)\right)$ is the inverse of a bivariate stable subordinator with index $\alpha \in(0,1)$ (defined in Subsection 2.2).

Then, for each $i=1,2, N_{i}\left(L_{i}(t)\right)$ is a fractional Poisson process, i.e. a counting renewal process with Mittag-Leffler inter-arrival times of type (4.13) (see e.g. [5], [6], [29], [22], [31] and also [7], [23], [28] for recent extensions). The discrete density $p_{i}(k, t)=P\left(N_{i}\left(L_{i}(t)\right)=k\right)$ is known to solve

$$
\frac{d^{\alpha}}{d t^{\alpha}} p_{i}(k, t)=-\xi_{i} p_{i}(k, t)+\xi_{i} p_{i}(k-1, t), \quad p_{i}(k, 0)=\delta_{0}(k) \quad k \in \mathbb{N}_{0},
$$

where the fractional derivative has been defined in (1.5).

Now, the process $\left(N_{1}\left(L_{1}^{\alpha}(t)\right), N_{2}\left(L_{2}^{\alpha}(t)\right)\right.$ is a bivariate renewal counting process in the sense of [14] and turns out to be an interesting bivariate generalization of the fractional Poisson process (other multivariate extensions had been recently proposed in [3] and [4]). In this case, equation (4.11) has the form

$$
\begin{aligned}
\mathcal{D}_{t_{1} t_{2}}^{\alpha} p\left(k_{1}, k_{2}, t_{1}, t_{2}\right) & =-\xi_{1} p\left(k_{1}, k_{2}, t_{1}, t_{2}\right)+\xi_{1} p\left(k_{1}-1, k_{2}, t_{1}, t_{2}\right) \\
& -\xi_{2} p\left(k_{1}, k_{2}, t_{1}, t_{2}\right)+\xi_{2} p\left(k_{1}, k_{2}-1, t_{1}, t_{2}\right), \quad k_{1}, k_{2} \in \mathbb{N}_{0},
\end{aligned}
$$

under the initial condition $p\left(k_{1}, k_{2}, 0,0\right)=\delta_{0}\left(k_{1}\right) \delta_{0}\left(k_{2}\right)$. Eq (4.15) is a generalization of (4.14). 


\section{CTRW LIMITS AND ANOMALOUS DIFFUSION IN ANISOTROPIC MEDIA}

Let $\left(B_{1}(t), B_{2}(t)\right)$ be a standard bi-dimensional Brownian motion and let $L^{\alpha}(t)$ be the inverse of an independent univariate stable subordinator of index $\alpha \in(0,1)$. The time-changed process

$$
\left\{\left(B_{1}\left(L^{\alpha}(t)\right), B_{2}\left(L^{\alpha}(t)\right)\right), t \geq 0\right\}
$$

has great importance in statistical physics, since it models the so-called anomalous diffusion (see, for example, [25], [26], [27], [37] and also [10] for recent developments). The term "anomalous" refers to the fact that the moving particle is subject to a sort of trapping effect which, in some sense, delays the time with respect to what happens for $\left(B_{1}(t), B_{2}(t)\right)$. Indeed, the mean square displacement grows as $t^{\alpha}$, i.e. slower with respect to the Brownian motion. Such a process is known to have a density $p\left(x_{1}, x_{2}, t\right)$ solving the equation

$$
\frac{\partial^{\alpha}}{\partial t^{\alpha}} p\left(x_{1}, x_{2}, t\right)=\frac{1}{2} \Delta p\left(x_{1}, x_{2}, t\right)
$$

where the operator on the left-hand side is the Caputo derivative (see (1.5)).

It is important to note that the above model assumes that the trapping effect is the same along both the coordinate directions, i.e. the time-changed process is isotropic as well as $\left(B_{1}(t), B_{2}(t)\right)$. Indeed, the time Laplace transform of the characteristic function of (5.1) has the form

$$
\int_{0}^{\infty} e^{-\eta t} \mathbb{E}\left[e^{i\left(\xi_{1} B_{1}\left(L^{\alpha}(t)\right)+\xi_{2} B_{2}\left(L^{\alpha}(t)\right)\right)}\right] d t=\frac{\eta^{\alpha-1}}{\eta^{\alpha}+\left(\xi_{1}^{2}+\xi_{2}^{2}\right) / 2}, \quad \xi_{1}, \xi_{2} \in \mathbb{R}, \eta \geq 0
$$

which is rotationally invariant in the variables $\xi_{1}, \xi_{2}$.

Here we present a model of motion in an anisotropic medium, such that the trapping effect depends on the coordinate direction. Let $\left(B_{1}(t), B_{2}(t)\right)$ be a standard bi-dimensional Brownian motion and let $\left(L_{1}^{\alpha}(t), L_{2}^{\alpha}(t)\right)$ be the inverse of a bivariate stable subordinator with Lévy measure $\phi_{\alpha}$ defined in (2.13); we construct the time-changed process

$$
\left\{\left(B_{1}\left(L_{1}^{\alpha}(t)\right), B_{2}\left(L_{2}^{\alpha}(t)\right)\right), t \geq 0\right\} .
$$

We remark that the trajectories of the models (5.1) and (5.3) have a different behaviour. In fact, in the isotropic case (5.1) the test particle stays at rest simultaneously in both directions at the intervals in which the random time $L^{\alpha}(t)$ has a plateaux, while in (5.3) the plateaux of $L_{1}^{\alpha}$ and $L_{2}^{\alpha}$ are not in general synchronized. For this reason we call this model anisotropic sub-diffusion. In conclusion, the motion for the second model (5.3) is given by the alternation of four different phases:

i) The particle is at rest in the $x$ direction but moves in the $y$ direction (when only $L_{1}^{\alpha}$ has a plateaux).

ii) The particle is at rest in the $y$ direction but moves in the $x$ direction (when only $L_{2}^{\alpha}$ has a plateaux).

iii) The particle stays at rest in both direction (when both $L_{1}^{\alpha}$ and $L_{2}^{\alpha}$ have a plateaux).

iv) The particle moves in both directions (otherwise).

Remark 5.1. Our model of anisotropic sub-diffusion is somewhat related to recent models of inhomogenous subdiffusion, which is governed by a time fractional 
equation of space-depending order

$$
\frac{\partial^{\alpha(x)}}{\partial t^{\alpha(x)}} p(x, t)=\frac{1}{2} \Delta p(x, t) \quad x \in \mathbb{R}^{d} .
$$

Such equation has been recently studied in [16], [17], [42] and [43]. In this model the intensity of the trapping effect depends on the space position rather than on the direction. In particular, in [42] and [43] the inhomogeneous subdiffusion is constructed by means of a particular time-change such that the inverse subordinator and the Brownian motion exhibit a suitable stochastic dependence.

According to Theorem 4.1, the random vector $\left(B_{1}\left(L_{1}\left(t_{1}\right)\right), B_{2}\left(L_{2}\left(t_{2}\right)\right)\right.$ has a density $p\left(x_{1}, x_{2}, t_{1}, t_{2}\right)$ which solves the equation

$$
\mathcal{D}_{t_{1}, t_{2}}^{\alpha} p\left(x_{1}, x_{2},, t_{1}, t_{2}\right)=\frac{1}{2} \Delta p\left(x_{1}, x_{2}, t_{1}, t_{2}\right) \quad\left(x_{1}, x_{2}\right) \in \mathbb{R}^{2} \backslash\{(0,0)\} ;
$$

this is a generalization of (5.2), where the operator on the left-hand side is defined in (2.16). The time-Laplace transform of the characteristic function can be computed by using (3.11) and a simple conditioning argument:

$$
\begin{aligned}
& \int_{0}^{\infty} \int_{0}^{\infty} e^{-\eta_{1} t_{1}-\eta_{2} t_{2}} \mathbb{E}\left[e^{i\left(\xi_{1} B_{1}\left(L_{1}\left(t_{1}\right)\right)+\xi_{2} B_{2}\left(L_{2}\left(t_{2}\right)\right)\right)}\right] d t_{1} d t_{2} \\
= & \int_{0}^{\infty} \int_{0}^{\infty} e^{-\eta_{1} t_{1}-\eta_{2} t_{2}} \mathbb{E}\left[e^{-\frac{1}{2} \xi_{1}^{2} L_{1}\left(t_{1}\right)-\frac{1}{2} \xi_{2}^{2} L_{2}\left(t_{2}\right)}\right] d t_{1} d t_{2} \\
= & \frac{\eta_{1}^{\alpha} \eta_{2}^{\alpha}}{\eta_{1} \eta_{2}\left[\xi_{1}^{2} / 2+\eta_{1}^{\alpha}\right]\left[\xi_{2}^{2} / 2+\eta_{2}^{\alpha}\right]}+ \\
& +\frac{\xi_{1}^{2} \xi_{2}^{2}\left[\eta_{1}^{\alpha}+\eta_{2}^{\alpha}-S_{\alpha}\left(\eta_{1}, \eta_{1}\right)\right]}{4 \eta_{1} \eta_{2}\left[\xi_{1}^{2} / 2+\eta_{1}^{\alpha}\right]\left[\xi_{2}^{2} / 2+\eta_{2}^{\alpha}\right]\left[\xi_{1}^{2} / 2+\xi_{2}^{2} / 2+S_{\alpha}\left(\eta_{1}, \eta_{1}\right)\right]},
\end{aligned}
$$

where $S_{\alpha}$ has been defined in (2.15).

5.1. Functional limit results. As said in the introduction, a well established theory (see e.g. [2], [34], [32], [33], [35], [47]) shows that (5.1) arises as CTRW scaling limit. For the reader's convenience we first report the main aspects of such a theory (in the particular case of bi-dimesional CTRW); then we prove that, by constructing another suitable class of CTRWs, also anisotropic subdiffusion (5.3) arises as a particular scaling limit.

Let $\left(Y_{i}^{1}, Y_{i}^{2}\right), i=1 \ldots, n$, be i.i.d. random vectors in $\mathbb{R}^{2}$ representing the jumps of a particle and let $S_{n}=\sum_{k=1}^{n}\left(Y_{k}^{1}, Y_{k}^{2}\right)$ be the discrete-time random walk giving the location of the particle after $n$ jumps, where $S_{0}=(0,0)$. Moreover, let $J_{i} \in \mathbb{R}^{+}$ be i.i.d. random variables representing the inter-arrival times between consecutive jumps and let $T_{n}=\sum_{k=1}^{n} J_{k}$ be the time of the $n$-th jump.

Let $N(t)=\max \left\{n \geq 0: T_{n} \leq t\right\}$ be a renewal counting process giving the number of jumps up to time $t \in \mathbb{R}^{+}$. A CTRW is defined as the time-changed process

$$
S_{N(t)}=\sum_{k=1}^{N(t)}\left(Y_{k}^{1}, Y_{k}^{2}\right), \quad t \in \mathbb{R}^{+},
$$

namely a process with jumps $\left(Y_{k}^{1}, Y_{k}^{2}\right)$ separated by inter-arrival times $J_{k}$. 
We now recall some functional limit results concerning the case of infinite mean inter-arrival times. Assume that the random variables $J_{i}$ belong to the domain of attraction of a positively skewed stable law with index $\alpha \in(0,1)$. Then

$$
c^{-\frac{1}{\alpha}} T_{[c t]} \rightarrow H^{\alpha}(t), \quad \text { as } c \rightarrow \infty
$$

in the $J_{1}$ topology, where $H^{\alpha}(t)$ is a $\alpha$-stable subordinator and $[x]$ denotes the biggest integer less than (or equal to) $x$. Moreover

$$
c^{-\alpha} N(c t) \rightarrow L^{\alpha}(t), \quad \text { as } c \rightarrow \infty
$$

in the $M_{1}$ topology, where $L^{\alpha}(t)=\inf \left\{x: H^{\alpha}(x)>t\right\}$ is an inverse $\alpha$ stable subordinator. Moreover, if the $\left(Y_{i}^{1}, Y_{i}^{2}\right)$ belong to the domain of attraction of a standard normal law, then $c^{-\frac{1}{2}} S_{[c t]}$ converges to a standard Brownian motion $\left(B_{1}(t), B_{2}(t)\right)$ in $J_{1}$ topology. Then, by combining the above results,

$$
c^{-\frac{\alpha}{2}} S_{N([c t])} \rightarrow\left(B_{1}\left(L^{\alpha}(t)\right), B_{2}\left(L^{\alpha}(t)\right) \quad \text { as } c \rightarrow \infty\right.
$$

in the $M_{1}$ topology, where the limit process is the subdiffusion defined by (5.1).

In the following subsection, we show how it is possible to construct a CTRW converging to the new process (5.3).

5.1.1. Limits of CTRWs driven by bivariate renewal processes. Let

$$
\left(J_{1}^{1}, J_{1}^{2}\right), \ldots,\left(J_{n}^{1}, J_{n}^{2}\right)
$$

be i.i.d. random vectors in $\mathbb{R}_{+}^{2}$ (such that, for each $i, J_{i}^{1}$ and $J_{i}^{2}$ are possibly dependent) and let $\left(T_{n}^{1}, T_{n}^{2}\right)$ be the simple random walk on $\mathbb{R}_{+}^{2}$ defined by

$$
\left(T_{n}^{1}, T_{n}^{2}\right)=\left(\sum_{k=1}^{n} J_{k}^{1}, \sum_{k=1}^{n} J_{k}^{2}\right) \quad n \in \mathbb{N}_{0} .
$$

Let $\left(N^{1}(t), N^{2}(t)\right)$ be a bivariate renewal counting process in the sense of [14], namely, for each $i=1,2$,

$$
N^{i}(t)=\max \left\{n \in \mathbb{N}_{0}: T_{n}^{i} \leq t\right\} \quad t \in \mathbb{R}^{+} .
$$

$N^{1}(t)$ and $N^{2}(t)$ are possibly dependent. Consider now another random walk $\left(S_{n}^{1}, S_{n}^{2}\right)$, defined by

$$
\left(S_{n}^{1}, S_{n}^{2}\right)=\left(\sum_{k=1}^{n} Y_{k}^{1}, \sum_{k=1}^{n} Y_{k}^{2}\right) \quad n \in \mathbb{N}_{0}
$$

where $\left(Y_{k}^{1}, Y_{k}^{2}\right), k=1, \ldots, n$, are i.i.d random vectors in $\mathbb{R}^{2}$.

We finally consider the CTRW defined by the bivariate time-change

$$
\left(S_{N^{1}(t)}^{1}, S_{N^{2}(t)}^{2}\right)=\left(\sum_{k=1}^{N^{1}(t)} Y_{k}^{1}, \sum_{k=1}^{N^{2}(t)} Y_{k}^{2}\right) \quad t \in \mathbb{R}^{+} .
$$

We note that the marginal components of (5.8) do not have simultaneous jumps (unlike the ones of (5.4)). Moreover, if considered separately, both $S_{N^{1}(t)}^{1}$ and $S_{N^{2}(t)}^{2}$ are CTRWs of the type already treated in the literature and then we can refer to the classical theory to perform their scaling limits.

The novelty of the following theorem is to present functional limit results in the case of bivariate time-change. We will denote by $\stackrel{J_{1}}{\rightarrow}\left(\right.$ resp. $\left.\stackrel{M_{1}}{\rightarrow}\right)$ the convergence in $J_{1}\left(\right.$ resp. $\left.M_{1}\right)$ topology on the space $D^{2}([0, \infty))$. 
Theorem 5.2. Let $\left(J_{k}^{1}, J_{k}^{2}\right)$ belong to the domain of attraction of a bivariate stable law with index $\alpha \in(0,1)$ and support on $\mathbb{R}_{2}^{+}$. Then

$$
c^{-\frac{1}{\alpha}}\left(T_{[c t]}^{1}, T_{[c t]}^{2}\right) \stackrel{J_{1}}{\rightarrow}\left(H_{1}^{\alpha}(t), H_{2}^{\alpha}(t)\right), \quad \text { as } c \rightarrow \infty
$$

and

$$
\left(c^{-\alpha} N_{1}(c t), c^{-\alpha} N_{2}(c t)\right) \stackrel{M_{1}}{\rightarrow}\left(L_{1}^{\alpha}(t), L_{2}^{\alpha}(t)\right), \quad \text { as } c \rightarrow \infty,
$$

where $\left(H_{1}^{\alpha}(t), H_{2}^{\alpha}(t)\right)$ is a bivariate stable subordinator (see Subsection 2.2) and $\left(L_{1}^{\alpha}(t), L_{2}^{\alpha}(t)\right)$ is its inverse (in the sense explained in section 3). Moreover, if the random vectors $\left(Y_{i}^{1}, Y_{i}^{2}\right)$ in (5.7) belong to the domain of attraction of a standard normal law, we have

$$
\left(c^{-\frac{\alpha}{2}} S_{N^{1}([c t])}^{1}, c^{-\frac{\alpha}{2}} S_{N^{2}([c t])}^{2}\right) \stackrel{M_{1}}{\rightarrow}\left(B_{1}\left(L_{1}^{\alpha}(t)\right), B_{2}\left(L_{2}^{\alpha}(t)\right) \quad \text { as } c \rightarrow \infty\right.
$$

where $\left(B_{1}(t), B_{2}(t)\right)$ denotes a standard Brownian motion.

Proof. Since $\left(J_{k}^{1}, J_{k}^{2}\right)$ belong to the domain of attraction of a bivariate $\alpha$-stable law, then, by Thm 6.21 at page 168 of [34], we have

$$
c^{-\frac{1}{\alpha}}\left(T_{[c t]}^{1}, T_{[c t]}^{2}\right) \stackrel{d}{\rightarrow}\left(H_{1}^{\alpha}(t), H_{2}^{\alpha}(t)\right) \quad \text { for all } t \geq 0, \quad \text { as } c \rightarrow \infty,
$$

where $\stackrel{d}{\rightarrow}$ denotes convergence in distribution. Then, by using [46], Thm. 2.7, condition (5.12) implies (5.9).

The bivariate renewal process $\left(N^{1}(t), N^{2}(t)\right)$ is a continuous functional in $M_{1}$ topology; then the continuous mapping argument provided by Thm. 1.6.13 in [45] (page 56) gives (5.10).

We finally observe that the set of discontinuities of the limit processes $\left(B_{1}(t), B_{2}(t)\right)$ and $\left(H_{1}^{\alpha}(t), H_{2}^{\alpha}(t)\right)$ are obviously disjoint as the Brownian motion has continuous sample paths; hence, another continuous mapping argument (i.e. a vector composition of processes in the sense of [45], Section 2.7) leads to (5.11).

Remark 5.3. Theorem 5.2 is restricted to the case where the inter-arrival times belong to the domain of attraction of a stable law, while the jumps belong to the domain of attraction of a normal distribution. We trust that a more general result could be obtained. Indeed, by using triangular array convergence, one could remove such assumptions and study the case where a rescaled CTRW converges to a Lévy process time-changed by the inverse of a general bivariate subordinator. However, in this case, there are some difficulties in treating simultaneous jumps of the components of the limit processes. So, a further analysis is needed on these points.

Acknowledgements. The authors thank the referee for his/her careful reading of the first version of the manuscript. Moreover they also thank Patrizia Semeraro for useful comments on her papers cited in the bibliography, Dmitrii Silvestrov for useful discussion on the $M_{1}$ convergence, and Fabrizio Durante for suggesting the references [12] and [13].

The authors acknowledge the support of GNAMPA-INdAM. Moreover C.M. acknowledges the support of MIUR Excellence Department Project awarded to the Department of Mathematics, University of Rome Tor Vergata (CUP E83C18000100006). 


\section{REFERENCES}

[1] O. Barndorff-Nielsen; J. Pedersen; K. Sato. Multivariate subordination, selfdecomposability and stability. Adv. in Appl. Probab. 33, no. 1, 160-187, 2001.

[2] P. Becker-Kern, M. M. Meerschaert, H. P. Scheffler. Limit theorems for coupled continuous-time random walks. Ann. Probab., 32, 730-756, 2004.

[3] L. Beghin; C. Macci. Asymptotic results for a multivariate version of the alternative fractional Poisson process. Statist. Probab. Lett. 129, 260-268, 2017.

[4] L. Beghin; C. Macci. Multivariate fractional Poisson processes and compound sums. Adv. in Appl. Probab. 48, no. 3, 691-711, 2016.

[5] L. Beghin, E. Orsingher. Fractional Poisson process and related planar motions. Electr. J. Prob., 14, 1790-1827, 2009.

[6] L. Beghin, E. Orsingher. Poisson-type processes governed by fractional higher order equations . Electr. J. Prob., 22, 684-709, 2010.

[7] L. Beghin, C. Ricciuti. Time inhomogeneous fractional Poisson processes defined by the multistable subordinator. Stoch. Anal. Appl., 37(2), 171-188, 2019.

[8] S. Bochner. Harmonic analysis and the theory of probability. University of California Press, Berkeley and Los Angeles, 1955.

[9] Bogdan, K., Byczkowski, T., Kulczycki, T., Ryznar, M., Song, R., Vondracek, Z.: Potential analysis of stable processes and its extensions. In: Graczyk, P., Stos, A (eds.) Lecture Notes in Mathematics 1980, 87-176, 2009.

[10] R. Capitanelli, M. D'Ovidio. Delayed and rushed motions through time-change. Alea, Lat. Am. J. Probab. Math. Stat., 17, 183-204, 2020.

[11] M. D'Ovidio.; R. Garra. Multidimensional fractional advection-dispersion equations and related stochastic processes. Electron. J. Probab. 19 , no. 61, 31 pp., 2014.

[12] H. Esmaeili, C. Klüppelberg. Parametric estimation of a bivariate stable Lévy process. J. Multivariate Anal. 102, no. 5, 918-930, 2011.

[13] H. Esmaeili, C. Klüppelberg. Two-step estimation of a multi-variate Lévy process. J. Time Series Anal. 34. no. 6, 668-690, 2013.

[14] J.J. Hunter. Renewal theory in two dimensions: basic results. Adv. Appl. Probab. Vol. 6, No. 2, 376-391, 1974.

[15] J. Kallsen, P. Tankov. Characterization of dependence of multidimensional Lévy processes using Lévy copulas. J. Multivariate Anal. 97, no. 7, 1551-1572, 2006.

[16] Y. Kian, D. Sambou, E. Soccorsi. Asymptotic estimates of solutions to time-fractional diffusion equations with space-dependent variable order. arXiv:1901.02958, 2019.

[17] Y. Kian, E. Soccorsi, M. Yamamoto. On time-fractional diffusion equations with space-dependent variable order, Ann. H. Poinc. 19(12), 3855-3881, 2018.

[18] Kilbas A.A., Srivastava H.M., Trujillo J.J., Theory and Applications of Fractional Differential Equations, vol. 204 of North-Holland Mathematics Studies, Elsevier Science B.V., Amsterdam, 2006.

[19] V.N. Kolokoltsov. Generalized Continuous-Time Random Walks, subordination by hitting times, and fractional dynamics. Theory Probab. Appl. 53, 594609, 2009.

[20] A. Kumar, A. Wylomanska, R. Poloczanski, J. Gajda, Fractional Brownian motion delayed by tempered and inverse tempered stable subordinators, Methodology and Computing in Applied Probability 21(1), 185-202, 2019. 
[21] A. Kumar, A. Wylomanska, R. Poloczanski, S. Sundar, Fractional Brownian motion time-changed by gamma and inverse gamma process, Physica A 468, 648-667, 2017.

[22] N. Laskin. Fractional Poisson process. Commun. Nonlinear Sci. Numer. Simul., 8 (2003), 201-213.

[23] N. N. Leonenko, E. Scalas, M. Trinh. The fractional non-homogeneous Poisson process. Statist. Probab. Lett., 120, 147-156, 2017.

[24] E. Luciano; P. Semeraro. Multivariate time-changes for Lévy asset models: characterization and calibration. J. Comput. Appl. Math. 233, no. 8, 19371953, 2010.

[25] M. Magdziarz, Path properties of subdiffusion: a martingale approach, Stochastic Models, 26, 256-271, 2010.

[26] M. Magdziarz, R. Schilling. Asymptotic properties of Brownian motion delayed by inverse subordinators. Proc. Am. Math. Soc 143, 4485-4501, 2015.

[27] M. Magdziarz; A. Weron. Ergodic properties of anomalous diffusion processes. Ann. Physics 326, no. 9, 2431-2443, 2011.

[28] A. Maheshwari, P. Vellaisamy. Non homogeneous space-time fractional Poisson process. Stoch. Anal. Appl., 37(2), 137-154, 2019.

[29] F. Mainardi, R. Gorenflo, E. Scalas. A fractional generalization of the Poisson processes. Vietnam Journ. Math. 32, 2004.

[30] M.M. Meerschaert, D. Benson, B. Baumer. Multidimensional advection and fractional dispersion. Physical Review E, Vol 59(5): 1-3, 1999.

[31] M.M. Meerschaert, E. Nane and P. Vellaisamy. The fractional Poisson process and the inverse stable subordinator. Elect. J. Prob., 16(59): 1600-1620, 2011.

[32] M.M. Meerschaert and H.P. Scheffler. Triangular array limits for continuous time random walks. Stoch. Proc. Appl., 118(9): 1606-1633, 2008.

[33] M.M. Meerschaert and H.P. Scheffler. Limit theorems for continuous-time random walks with infinite mean waiting times. J. Appl. Probab., 41: 623-638, 2004.

[34] M.M. Meerschaert, A. Sikorskii. Stochastic models for fractional calculus. De Gruyter Studies in Mathematics, 43. Walter de Gruyter Co., Berlin, 2012.

[35] M.M. Meerschaert and P. Straka. Semi-Markov approach to continuous time random walk limit processes. Ann. Probab., 42(4) : 1699-1723, 2014.

[36] M.M. Meerschaert, B. Toaldo. Relaxation patterns and semi-Markov dynamics. Stoch. Proc. Appl., 129(8), 2850-2879, 2019.

[37] R. Metzler, J. Klafter: The random walk's guide to anomalous diffusion: a fractional dynamics approach. Phys. Rep. 339, 1-77, 2000.

[38] H. Ogasawara. The multivariate Markov and multiple Chebyshev inequalities, Comm. Stat. Theory and Methods, in press, 2019.

[39] J. Pedersen; K. Sato, Cone-parameter convolution semigroups and their subordination. Tokyo J. Math. 26, no. 2, 503-525, 2003.

[40] J. Pedersen; K. Sato, Relations between cone-parameter Lévy processes and convolution semigroups. J. Math. Soc. Japan 56, no. 2, 541-559, 2004.

[41] J. Pedersen; K. Sato, Semigroups and processes with parameter in a cone. Abstract and applied analysis, 499-513, World Sci. Publ., River Edge, NJ, 2004.

[42] C. Ricciuti; B. Toaldo. Semi-Markov models and motion in heterogeneous media. J. Stat. Phys. 169, no. 2, 340-361, 2017. 
[43] M. Savov, B. Toaldo, Semi-Markov processes, integro-differential equations and anomalous diffusion-aggregation, Annales de l'Institut Henri Poincar (B) Probability and Statistics, 2020, in press.

[44] P. Semeraro. A multivariate variance gamma model for financial applications. Int. J. Theor. Appl. Finance 11, no. 1, 1-18, 2008.

[45] D.S. Silvestrov. Limit Theorems for Randomly Stopped Stochastic Processes, Springer, 2004.

[46] A. V. Skorohod. Limit theorems for stochastic processes with independent increments, Theory Probab. Appl., 2, 138-170, 1957.

[47] P. Straka, B.I. Henry, Lagging and leading coupled continuous time random walks, renewal times and their joint limits, Stoch. Proc. Appl. 121 324-336, 2011. 